\title{
Spectral Estimates \\ for Compact Hyperbolic Space Forms and the \\ Selberg Zeta Function for $p$-Spectra II
}

\author{
R. Schuster
}

\begin{abstract}
We prove an asymptotic estimation for the spectrum of the Laplace operator for compact hyperbolic space forms. Thereby we use estimations of the Selberg zeta function by methods of analytic number theory.
\end{abstract}

Keywords: Eigenvalue spectrum, Laplace operator, hyperbolic space form, length spectrum, Selberg zeta function, analytic number theory techniques

AMS subject classification: 11F72, 11M06, 35Q05, 58F19, 58F25

\section{Introduction}

First we recall the main definitions, motivations and results of Part I. Let $\mathcal{G}$ be a properly discontinuous group of orientation preserving isometries of the $n$-dimensional hyperbolic space $\mathbf{H}_{n}$ of constant curvature -1 without fixed points (with the exception of the identity map id) with compact fundamental domain. We consider the related Killing-Hopf space form $V=H_{n} / \mathcal{G}$. Let $\Omega$ be the set of non-trivial free homotopy classes of $V$. In every class $\omega \in \Omega$ there lies exactly one closed geodesic line. We denote by $l(\omega)$ and $\nu(\omega)$ its length and muliplicity, respectively. The parallel displacement along a closed geodesic line induces an isometry of the tangent space in every point of that geodesic line with the eigenvalues $\beta_{1}(\omega), \ldots, \beta_{n-1}(\omega), 1$ with $\left|\beta_{i}(\omega)\right|=1(i=1, \ldots, n-1)$. Let $e_{p}(\omega)$ be the $p^{\text {th }}$ elementary symmetric function of the $\beta_{i}(\omega)(i=1, \ldots, n-1)$, and put $e_{0}(\omega)=1$. Further on, we introduce the weight

$$
\sigma(\omega)=\frac{e^{N l(\omega)}}{\nu(\omega)} \prod_{j=1}^{n-1} \frac{1}{\left(e^{l(\omega)}-\beta_{j}(\omega)\right)}
$$

with $N=(n-1) / 2$. Let $S_{p}$ denote the p-spectrum of the Laplace operator $\Delta=d \delta+\delta d$.

R. Schuster: Univ. Leipzig, Institut für Mathematik, Augustusplatz 10, D - 04109 Leipzig

ISSN 0232-2064 / $\$ 2.50$

(C) Heldermann Verlag Berlin 
Thereby we have used the differential operatord and the codifferential operator $\delta=$ $(-1)^{p n+n-1} * d *$ for differential $p$-forms, where * denotes the Hodge dualization. Let $d_{p}^{d}(\mu)$ and $d_{p}^{\delta}(\mu)$ denote the dimension of the eigenspaces of closed $(d \alpha=0)$ and coclosed $(\delta \alpha=0)$ eigenforms $\alpha$ of $\Delta$ with eigenvalues $\mu$, respectively. The dimension of the space of harmonic $p$-eigenforms is the $p^{\text {th }}$ Betty number $B_{p}$ of the space form $V$.

Our results are based on the Selberg trace formula as a duality statement between the p-eigenvalue spectrum and the geometric spectrum of $V$ (expressed by $l(\omega), \nu(\omega), \sigma(\omega)$ and $\left.e_{p}(\omega)\right)$.

Theorem 1 (Selberg trace formula): Let $h=h(r)$ be an analytic function in the strip $\mid$ Im $r \mid<N+\delta$ with $N=(n-1) / 2,0<\delta<1 / 2$, which is even, $h(r)=$ $h(-r)$, and satisfies $|h(r)| \leq A(1+|r|)^{-n-\delta}$. By the help of the Fourier transform $g(u)=$ $\frac{1}{2 \pi} \int_{-\infty}^{\infty} h(r) e^{-i r u} d r$ of $h$ we can state the trace formula

$$
\sum_{\mu \in S_{p}} d_{p}^{*}(\mu) h\left(r_{p}(\mu)\right)=\operatorname{vol} V\left\langle S_{p}^{n}, g\right\rangle+\sum_{\omega \in \Omega} l(\omega) \sigma(\omega) e_{p}(\omega) g(l(\omega))
$$

for $p=0, \ldots, n-1$ with $r_{p}(\mu)=\sqrt{\mu-(p-N)^{2}}$, where

$$
\left\langle S_{p}^{n}, g\right\rangle=\frac{2\left(\begin{array}{c}
n-1 \\
p
\end{array}\right)}{(4 \pi)^{n / 2} \Gamma(n / 2)} \begin{cases}\int_{0}^{\infty}\left(\prod_{\substack{n=0 \\
u \neq|p-N|}}^{N}\left(r^{2}+u^{2}\right)\right) h(r) d r & \text { for } n \text { odd } \\
\int_{0}^{\infty}\left(\prod_{\substack{u=1 / 2 \\
u \neq|p-N|}}^{N}\left(r^{2}+u^{2}\right)\right) h(r) r \tanh (\pi r) d r & \text { for } n \text { even. }\end{cases}
$$

Thereby we have used $N=\frac{n-1}{2}$ and

$$
d_{p}^{*}(\mu)= \begin{cases}d_{p}^{\delta}(\mu) & \text { for } \mu>0 \\ (-1)^{p}\left(B_{0}-B_{1}+\ldots+(-1)^{p} B_{p}\right)+K_{p} & \text { for } \mu=0\end{cases}
$$

with

$$
K_{p}= \begin{cases}(-1)^{p+1-n / 2} \pi^{-(n+1) / 2} \Gamma\left(\frac{n+1}{2}\right) \text { vol } V & \text { for } p \geq n / 2 \text { ( } n \text { even) } \\ 0 & \text { for the other cases. }\end{cases}
$$

Further on, vol $V$ denotes the volume of the space form $V$. theorem.

For $a>0$ we define $E(t, a)=\int_{1}^{t} e^{a s} / s d s$. The main result of Part I was the following

Theorem A: We can estimate the sum

$$
P_{p}(T)=\sum_{\substack{\omega \in \mathbb{2} \\ \cos (1(\omega) \leq T}} e_{p}(\omega) \quad \text { for } T \rightarrow \infty
$$


by

$$
P_{p}(T)=\left\{\begin{array}{cc}
O\left(T^{\left.N+\frac{(n-1)^{2}}{2 n} / \ln T\right)}\right. & \text { for } 1 \leq p \leq n-2 \\
E(t, n-1)+\sum_{\substack{\mu \in S_{p} \\
0<\mu<N^{2}(2 n-1) / n^{2}}} d_{p}^{\delta}(\mu) E\left(t, N+\sqrt{N^{2}-\mu}\right) & \\
+O\left(T^{\left.N+\frac{(n-1)^{2}}{2 n} / \ln T\right)}\right. & \text { for } p=0, n-1 .
\end{array}\right.
$$

with $N=\frac{n-1}{2}, \cosh t=T, T>1$. We get the same estimation if we replace $P_{p}(T)$ by

$$
P_{p}^{\#}(T)=\sum_{\substack{\cosh (\omega) \leq T, \mu(\omega)=1 \\((\omega)}} e_{p}(\omega) .
$$

In Part I we have proved Theorem A using a Landau difference method and a solution of an Euler-Poisson-Darboux equation (cf. Section 3 in Part I) as a special function which we can use in the Selberg trace formula. In some cases these functions are better adapted to the geometric situation than functions which are usually taken in trace formulas when a Selberg zeta function is considered.

In Section 6 we will introduce a Selberg zeta function in a natural way with respect to our version of the Selberg trace formula. This zeta function is well known for the case $n=2, p=0$. Gangolli [9] treats zeta functions of Selberg's type for compact space forms of symmetric spaces of rank one from the viewpoint of representation theory. To see differences to our treatment one should compare the zeros and poles of the analytic continuation of the zeta function to the whole complex plane. The Selberg trace formula bears a striking resemblance to the explicite formulas of prime number theory. The Selberg zeta function is analogous in many ways to the classical Riemann zeta function. This enables us to study the asymptotic behaviour of the p-spectrum using techniques of analytic number theory. As a consequence of the well-known Weyl type asymptotic formula (cf. $[2,28]$ and Section 4) we have

$$
\mathcal{N}_{p}(T)=\sum_{\substack{\mu \in S_{p} \\ \mu<T^{2}+(p-N)^{2}}} d_{p}^{6}(\mu) \sim n_{p} T^{n} \quad \text { for } T \rightarrow \infty
$$

with

$$
n_{p}=\frac{\left(\begin{array}{c}
n-1 \\
p
\end{array}\right) \text { vol } V}{(4 \pi)^{n / 2} \Gamma\left(\frac{n+2}{2}\right)} \quad \text { and } \quad N=\frac{n-1}{2} .
$$

We will prove the following

Theorem B: The error term $\mathcal{R}_{p}(T)$ defined by $\mathcal{N}_{p}(T)=n_{p} T^{n}+\mathcal{R}_{p}(T)(T>1)$ satisfies $\left|R_{p}(T)\right|=O\left(T^{n-1} / \ln T\right)$.

Hejhal [14] has given this estimation in the case $n=2, p=0$. Weaker results for more general spaces were proved by Gangolli [8] and Ivrii [17] for $n \geq 2, p=0$. Hejhal [14] remarked (for $n=2$ ) that it seems hard to improve the estimation of Theorem $B$. 
The analogy between the Selberg and the Riemann zeta function is strongly apparent in our proofs. If one were able to improve the $T^{n-1} / \ln T$ - term in Theorem $B$, there would presumably be a corresponding improvement in the estimation $\arg \zeta(1 / 2+i T)=$ $O(\ln T / \ln \ln T)$ for the Riemann zeta function, assuming the Riemann hypothesis is valid. But no such improvement is known.

Section 7 deals with the analytic continuation of the Selberg zeta funktion and its logarithmic derivative. We state funtional equations for these functions. In Section 8 we give an estimation for the Selberg zeta function based on the Weyl estimation for the eigenvalue spectrum. We use the method of "good" and "admissible" numbers (cf. Hejhal [14] and Ingham [16]), but a straightforward generalization to the $n$-dimensional case would not be strong enought. In Section 9 we prove spectral estimations based on estimations for the zeta functions. We use these results in Section 10 in order to derive estimations for the logarithmic derivative of the Selberg zeta function. We essentially use these estimations to prove Theorem B in Section 11.

\section{Definition of the Selberg zeta function and first properties}

Since we have already noted that the Selberg trace formula (Theorem 1 in Part 1 ) bears a striking resemblance to the so-called explicit formulas of prime number theory, it is quite natural to search for a function which is analogous to the classical Riemann zeta function. Nowadays this function $Z$ is commonly known as the Selberg zeta function, the original reference is [24] (Part I). We generalize a method given by Hejhal [14] for the classical case $n=2, p=0$. In this special case the Selberg zeta funtion is defined by

$$
Z(s)=\prod_{\substack{\omega \in \Omega \\ \eta(\omega)=1}} \prod_{m=0}^{\infty}\left(1-e^{-l(\omega)(\bullet+m)}\right)
$$

for $R e s>1$. Elementary calculations show that $\frac{d}{d s} \ln Z(s)$ is the right-hand side of the Selberg trace formula in the version of Theorem 1 (Part I) with $g(u)=e^{-u(s-1 / 2)}$. In the general case we start with the definition of a function $\Psi_{p}$ which later on (cf. Lemma 13) will be identified to be the logarithmic derivative of a Selberg zeta function $Z_{p}$. We define

$$
\Psi_{p}(s)=\kappa_{p} \sum_{\omega \in \Omega} l(\omega) \sigma(\omega) e_{p}(\omega) e^{-l(\omega)(o-N)}
$$

for $\operatorname{Res}>2 N$ with $N=(n-1) / 2\left(\right.$ as in Part I), $\kappa_{p}=1$ for $n$ odd and $\kappa_{p}=\Gamma(p+1) \Gamma(n-p)$ for $n$ even.

Lemma 12: The series (62) converges absolutely and uniformly for $s$ in the half space $R e s \geq 2 N+\epsilon_{0}, \epsilon_{0}>0$.

Proof: As a consequence of Proposition 5 we get

$$
\left|G_{p, 0}(T)\right|=\left|\sum_{\substack{\omega \in \Omega \\ \cosh (\omega) \leq T}} e_{p}(\omega) l(\omega) \sigma(\omega)\right|=O\left(T^{N}\right) .
$$


Using Stieltjes integration, we immediately get the assertion -

We denote the $j^{\text {th }}$ power of the eigenvalues $\beta_{i}(\omega)$ given by the parallel displacement along the closed geodesic line (cf. Introduction to Part I) belonging to a non-trivial free homotopy class $\omega \in \Omega$ by $\beta_{i}^{j}(\omega)=\left(\beta_{i}(\omega)\right)^{j}$. We can write the elementary symmetric function $e_{p}$ as a sum

$$
e_{p}(\omega)=\sum_{k=1}^{q} \epsilon_{p, k}(\omega) \text { with } q=\left(\begin{array}{c}
n-1 \\
p
\end{array}\right) \text { and } \epsilon_{p, k}(\omega)=\beta_{i_{1}}(\omega) \ldots \beta_{i_{p}}(\omega)
$$

using $i_{1}<i_{2}<\ldots<i_{p}$ and $i_{1}, i_{2}, \ldots, i_{p} \in\{1,2, \ldots, n-1\}$. Let $M$ denote the set of multiindices $m=\left(m_{1}, \ldots, m_{n-1}\right)$ with non-negative integers $m_{i}$. Further on we define in the usual way $\beta^{m}(\omega)=\beta_{1}^{m_{1}}(\omega) \ldots \beta_{n-1}^{m_{n-1}}(\omega)$ and $|m|=m_{1}+\ldots+m_{n-1}$.

Lemma 13: The zeta function

$$
Z_{p}(s)=\prod_{\substack{\omega \in \mathbb{\Omega} \\(\omega)=1}} \prod_{k=1}^{q} \prod_{m \in \mathbb{M}}\left(1-\epsilon_{p, k}(\omega) \beta^{m}(\omega) e^{-((\omega)(\iota+|m|)}\right)^{\kappa_{p}}
$$

satisfies

$$
\Psi_{p}(s)=\frac{d}{d s} \ln Z_{p}(s) .
$$

The product on the right-hand side of (63) is absolutely convergent for Re $s>2 N$. We have $Z_{p}(\bar{s})=\overline{Z_{p}(s)}, \Psi_{p}(\bar{s})=\overline{\Psi_{p}(s)}$, where $\bar{s}$ denotes the complex conjugate number of $s$.

Proof: The product on the right-hand side of (63) is absolutely convergent, if the series

$$
\begin{aligned}
& \sum_{\substack{\omega \in \Omega \\
\mu(\omega)=1}} \sum_{k=1}^{q} \sum_{m \in M}\left|\epsilon_{p, k}(\omega)\right|\left|\beta^{m}(\omega)\right| e^{-l(\omega)(R e s+|m|)} \\
& =\sum_{\substack{s \in \mathbb{R} \\
(\omega)=1}} \sum_{k=1}^{q} \sum_{m \in M} e^{-l(\omega)(\text { Rest }+m \mid)} \\
& =q \sum_{\substack{\omega \in \Omega \\
(\omega)=1}}\left(\prod_{j=1}^{n-1} \frac{1}{1-e^{-l(\omega)}}\right) e^{-l(\omega) R e .}
\end{aligned}
$$

is convergent. Thereby we have used $\left|\epsilon_{p, k}(\omega)\right|=1,\left|\beta^{m}(\omega)\right|=1$. It is sufficient to prove the convergence of $\sum_{\substack{\omega \in n \\(\omega)=1}} e^{-l(\omega) R e s}$ for $R e s>2 N$, which is a consequence of Proposition 5 (for $p=0$ ) using Stieltjes integration. Using (3) and the fact that every free homotopy class is the power of a primitive homotopy class, we can write

$$
\Psi_{p}(s)=\kappa_{p} \sum_{\substack{\omega \in \Omega \\(\omega)=1}} \sum_{j=1}^{\infty} l(\omega) e_{p}\left(\omega^{j}\right)\left(\prod_{i=1}^{n-1} \frac{1}{1-\beta_{i}^{j}(\omega) e^{-l(\omega) j}}\right) e^{-l(\omega) j} .
$$

Using the definitions, we get

$$
e_{p}\left(\omega^{j}\right)=\sum_{k=1}^{q} \epsilon_{p, k}^{j}(\omega) \text { with } \epsilon_{p, k}^{j}(\omega)=\left(\epsilon_{p, k}(\omega)\right)^{j} .
$$


and thereby it follows

$$
\Psi_{p}(s)=\kappa_{p} \sum_{\substack{\omega \in n \\(\omega)=1}} \sum_{j=1}^{\infty} \sum_{k=1}^{q} l(\omega) \epsilon_{p, k}^{j}(\omega)\left(\prod_{i=1}^{n-1} \frac{1}{1-\beta_{i}^{j}(\omega) e^{-l(\omega) j}}\right) e^{-l(\omega) j \cdot}
$$

Applying geometric series we get

$$
\prod_{i=1}^{n-1} \frac{1}{1-\beta_{i}^{j}(\omega) e^{-l(\omega) j}}=\sum_{m_{1}=0}^{\infty} \ldots \sum_{m_{n-1}=0}^{\infty} e^{-j\left(m_{1}+\ldots+m_{n-1}\right) l(\omega)} \prod_{i=1}^{n-1} \beta_{i}^{j m_{i}}(\omega) .
$$

It follows

$$
\begin{aligned}
\Psi_{p}(s) & =\kappa_{p} \sum_{\substack{\omega \in \Omega \\
(\omega)=1}} \sum_{j=1}^{\infty} \sum_{k=1}^{q} \sum_{m \in M} l(\omega) \epsilon_{p, k}^{j}(\omega) \beta^{j m}(\omega) e^{-l(\omega) j(s+|m|)} \\
& =\kappa_{p} \sum_{\substack{\omega \in \in \Omega \\
(\omega)=1}} \sum_{k=1}^{q} \sum_{m \in M} l(\omega) \frac{\epsilon_{p, k}(\omega) \beta^{m}(\omega) e^{-l(\omega)(\bullet+|m|)}}{1-\epsilon_{p, k}(\omega) \beta^{m}(\omega) e^{-l(\omega)(\bullet+|m|)}} .
\end{aligned}
$$

This proves (64)

Lemma 14: Using $s=\sigma^{*}+i T$, it holds

(i) $\left|\ln Z_{p}(s)\right| \leq c^{*} \sum_{\omega \in \Omega, \nu(\omega)=1} e^{-l(\omega) \sigma^{*}}$ for $\sigma^{*}>n-1$ with $c^{*}=\kappa_{p}\left(\begin{array}{c}n-1 \\ p\end{array}\right)\left(1-e^{-l_{0}}\right)^{-n}$ and $l_{0}=\inf _{\omega \in \Omega}\{l(\omega)\}$

(ii) $\ln Z_{p}(s)=O\left(e^{-l_{0} \sigma^{*}}\right)$ uniformly for $\sigma^{*}>n-1+\epsilon, 0<\epsilon<1,|s| \rightarrow \infty$

(iii) $Z_{p}(s)=1+O\left(e^{-10 \sigma^{*}}\right)$ uniformly for $\sigma^{*}>n-1+\epsilon, 0<\epsilon<1,|s| \rightarrow \infty$.

Proof: Definition (63) guarantees $R e Z_{p}(s)>0$ for Res $>n-1$. We use the branch of $\ln Z_{p}(s)$ with $-\pi \leq I m \ln Z_{p}(s)<\pi$ for $R e s>n-1$. The proof is a straightforward generalization of Hejhal [14, Proposition 4.13] using Proposition A (Part I), Lemma 12 and Lemma 13. For more details cf. Schuster [35]

As a direct consequence from the definition (62) and Theorem $A$ we get

Lemma 15: Let $s=\sigma^{*}+i T$. Then it holds $\left|\Psi_{p}(s)\right|=O(1)$ uniformly for $\sigma^{*} \geq$ $n-1+\epsilon, \epsilon>0,|s| \rightarrow \infty$. - 33]).

An other version of a Selberg zeta function is given by Christian [29, 30] (cf. also [31

\section{Analytic continuation of the Selberg zeta function}

Theorem 1 will come in handy if we try to find an analytic continuation of the Selberg zeta function. Till now we have only used the right-hand side of the Selberg trace formula for the definition of the logarithmic derivative of that zeta funtion. We will apply standard 
analytic number theory techniques which already have been used by Hejhal [14] in the classical case $n=2, p=0$. It is possible to carry this idea through, but we will have much more technical problems.

Using $\alpha_{i} \neq \alpha_{j}$ for $i \neq j(i, j=1, \ldots, m), m>n / 2$ we define

$$
\begin{aligned}
\mathcal{P}(i, s) & =\prod_{\substack{j=2 \\
j \neq i}}^{m}\left(\alpha_{j}^{2}-s^{2}\right), \quad \mathcal{P}^{+}(i, s)=\prod_{\substack{j=2 \\
j \neq i}}^{m}\left(\alpha_{j}^{2}+s^{2}\right), \\
\mathcal{Q}(i, s) & =\prod_{\substack{j=1 \\
j \neq i}}^{m}\left(\alpha_{j}^{2}-s^{2}\right), \quad \mathcal{Q}^{+}(i, s)=\prod_{\substack{j \neq 1 \\
j \neq i}}^{m}\left(\alpha_{j}^{2}+s^{2}\right), \\
\mathcal{R}(s) & =\prod_{j=1}^{m}\left(\alpha_{j}^{2}+s^{2}\right) .
\end{aligned}
$$

An empty product shall have the value 1 . From now on we use the following

Assumption 16: We use $m=\frac{n}{2}+1$ for $n$ even and $m=\frac{n+1}{2}$ for $n$ odd. Put $\alpha_{1}=s-N=\sigma+i T, N=(n-1) / 2$. We suppose $\alpha_{i} \in \mathbf{R}$ for $i=2, \ldots, m$, and $1+N<\alpha_{2}<\alpha_{3}<\ldots<\alpha_{m}$. Futher on we suppose $\alpha_{i} \neq N+k$ for $i=2, \ldots, m, k \in \mathbf{Z}$ and $\alpha_{1} \neq \alpha_{i}$ for $i=2, \ldots, m$.

We apply the Selberg trace formula with the function

$$
h^{\#}(r)=\frac{1}{\mathcal{R}(r)}
$$

Then the assumptions of Theorem 1 are fulfilled for $R e s \geq 2 N+\epsilon_{0}, \epsilon_{0}>0$. Gangolli [9] takes another special function (and another version of the trace formula) using a cut-off function. The resulting formulas are not explicite enough to use those methods of analytic number theory which we will apply. By partial fraction expansion we get

Lemma 17: We suppose $\alpha_{i} \neq \alpha_{j}$ for $i \neq j(i, j=1, \ldots, m)$. Then we get

$$
\frac{1}{\mathcal{R}(r)}=\sum_{j=1}^{m} \frac{1}{\mathcal{Q}\left(j, \alpha_{j}\right)} \frac{1}{r^{2}+\alpha_{j}^{2}} .
$$

We remark, that the summands in (69) allone do not fulfill the growth assumption of Theorem 1. We easily obtain the Fourier transform.

$$
g^{\#}(u)=\sum_{j=1}^{m} \frac{1}{\mathcal{Q}\left(j, \alpha_{j}\right)} \frac{e^{-\alpha_{j}|u|}}{2 \alpha_{j}}
$$

of $h^{\#}(r)=\frac{1}{\mathcal{R}(r)}$ (cf. [14] for $\mathrm{n}=2, \mathrm{~m}=2$ ). We define (cf. Part I) $r_{p}(\mu)=\sqrt{\mu-(N-p)^{2}}$ for $\mu \geq(N-p)^{2}$ and $r_{p}(\mu)=\sqrt{(N-p)^{2}-\mu}$ i for $\mu<(N-p)^{2}$ with the imaginary unit i. 
Proposition 18: The logarithmic derivative

$$
\Psi_{p}(s)=\kappa_{p} \sum_{\omega \in \Omega} l(\omega) \sigma(\omega) e_{p}(\omega) e^{-l(\omega)(\varepsilon-N)}
$$

of the Selberg zeta function

$$
Z_{p}(s)=\prod_{\substack{\omega \in \Omega \\(\omega)=1}} \prod_{k=1}^{q} \prod_{m \in M}\left(1-\epsilon_{p, k}(\omega) \beta^{m}(\omega) e^{-l(\omega)(e+|m|)}\right)^{\kappa_{p}}
$$

satisfies the equation

$$
\begin{aligned}
\frac{\Psi_{p}(s)}{2(s-N) \kappa_{p}}= & \sum_{i=2}^{m} \frac{\mathcal{P}(i, s-N)}{\mathcal{P}\left(i, \alpha_{i}\right)} \frac{\Psi_{p}\left(\alpha_{i}+N\right)}{2 \alpha_{i} \kappa_{p}} \\
& +\sum_{\mu \in S_{p}} d_{p}^{*}(\mu) \frac{\mathcal{Q}(1, s-N)}{\mathcal{R}\left(r_{p}(\mu)\right)}-\left\langle S_{p}^{n}, g^{\#}\right\rangle \mathcal{Q}(1, s-N) \text { vol } V
\end{aligned}
$$

for $\sigma>N$ supposing Assumption 16.

Proof: The functions $h^{\#}(r)$ and $g^{\#}(u)$ satisfy the assumptions of Theorem 1 if we suppose Assumption 16 using $\frac{\mathcal{Q}(1, \varepsilon-N)}{\mathcal{Q}\left(j, \alpha_{j}\right)}=-\frac{\mathcal{P}(j, s-N)}{\mathcal{P}\left(j, \alpha_{j}\right)}$. The application of the Selberg trace formula (5) and Lemma 17 proves the proposition

We remark that we can not split up the sum $\sum_{\mu \in S_{p}}$ in (71) using (69) as we have done it for $\Psi_{p}$ using (70) because of the fact that we have to guarantee the convergence of the sum $\sum_{\mu \in S_{p}}$. Next we are interested in the meromorphic continuation of that sum. For $\operatorname{Res}>n-1$ we define

$$
\mathcal{A}(s)=\sum_{\mu \in S_{p}} d_{p}^{*}(\mu) \frac{2(s-N) \mathcal{Q}(1, s-N)}{\mathcal{R}\left(r_{p}(\mu)\right)} .
$$

Standard considerations $(c f .[9,14])$ give

Lemma 19: The function $\mathcal{A}(s)$ has a meromorphic continuation into the whole complex plane. The poles are $s^{+}(\mu)=N+i r_{p}(\mu)$ and $s^{-}(\mu)=N-i r_{p}(\mu)$ for $\mu \in S_{p}$ and $d_{p}^{*}(\mu) \neq 0$. These are simple poles with residues $d_{p}^{*}(\mu)$ if $\mu \neq(N-p)^{2}$ and with residue $2 d_{p}^{*}(\mu)$ if $\mu=(N-p)^{2}$.

In order to get the meromorphic continuation of $\Psi_{p}(s)$ we define

$$
\mathcal{W}(s)=-\mathcal{Q}(1, s-N)\left\langle S_{p}^{n}, g^{\#}\right\rangle \text { vol } V
$$

for $\operatorname{Re} s>2 N$. We start with the discussion of the case of $n$ even. Using (6) and (22) we a]so can write

$$
\mathcal{W}(s)=c_{W} \int_{-\infty}^{+\infty} r \bar{h}(r) \tanh (\pi r) d r
$$


with

$$
\bar{h}(r)=P\left(r^{2}\right) \frac{\mathcal{Q}(1, s-N)}{\mathcal{R}(r)}, P(s)=\prod_{\substack{s=1 / 2 \\ s \neq 1 / N-p 1}}^{N}\left(s+u^{2}\right), c_{w}=\frac{(-1)^{\frac{n-2}{2}} \chi(V)}{2 \Gamma(p+1) \Gamma(n-p)} .
$$

The polynomial $P$ is of degree $n / 2-1=m-2$.

Lemma 20: For $n$ even the function $\mathcal{W}(s)=-\mathcal{Q}(1, s-N)\left(S_{p}^{n}, g^{\#}\right)$ vol $V$ has a meromorphic continuation into the whole complez plane which is given by

$$
\begin{aligned}
\frac{W(s)}{c_{W}}= & P\left(-(s-N)^{2}\right) \frac{-2|N-p|}{-(N-p)^{2}+(s-N)^{2}} \\
& -\sum_{j=2}^{m} P\left(-\alpha_{j}^{2}\right) \frac{\mathcal{P}(j, s-N)}{\mathcal{P}\left(j, \alpha_{j}\right)} \frac{-2|N-p|}{-(N-p)^{2}+\alpha_{j}^{2}} \\
& +\sum_{k=1}^{\infty}\left(P\left(-(s-N)^{2}\right) \frac{-2|N+k|}{-(N+k)^{2}+(s-N)^{2}}\right. \\
& \left.-\sum_{j=2}^{m} P\left(-\alpha_{j}^{2}\right) \frac{\mathcal{P}(j, s-N)}{\mathcal{P}\left(j, \alpha_{j}\right)} \frac{-2|N+k|}{-(N+k)^{2}+\alpha_{j}^{2}}\right) \\
& -P\left(-(s-N)^{2}\right) \pi \tan (\pi(s-N))+\sum_{j=2}^{m} P\left(-\alpha_{j}^{2}\right) \frac{\mathcal{P}(j, s-N)}{\mathcal{P}\left(j, \alpha_{j}\right)} \pi \tan \left(\pi \alpha_{j}\right) .
\end{aligned}
$$

The function $\mathcal{W}(s)$ has simple poles in the points $s=N-|N-p|$ and $s=-k(k \in \mathbf{N})$ with the residues $2 c_{W} P\left(-(N-p)^{2}\right)$ and $2 c_{W} P\left(-(N+k)^{2}\right)$, respectively. In the other points there is holomorphic behaviour.

Proof: Clearly we obtain

$$
\frac{P\left(r^{2}\right)}{\mathcal{R}(r)}=\sum_{j=1}^{m} \frac{P\left(-\alpha_{j}^{2}\right)}{\mathcal{Q}\left(j, \alpha_{j}\right)} \frac{1}{r^{2}+\alpha_{j}^{2}}
$$

It follows

$$
\bar{h}(r)=P\left(-\alpha_{1}^{2}\right) \frac{1}{r^{2}+\alpha_{1}^{2}}-\sum_{j=2}^{m} P\left(-\alpha_{j}^{2}\right) \frac{\mathcal{P}\left(j, \alpha_{1}\right)}{\mathcal{P}\left(j, \alpha_{j}\right)} \frac{1}{r^{2}+\alpha_{j}^{2}} .
$$

Thereby we have used $\alpha_{1}=s-N$. In order to calculate the integral $\int_{-\infty}^{\infty} r \bar{h}(r) \tanh (\pi r) d r$ we apply the residue theorem. The convergence of the sum $\sum_{k=1}^{\infty}$ in (76) is guaranteed by the convergence of $\sum_{k=1}^{\infty}(N+k) \bar{h}(|N+k| i)=O\left(\sum_{k=1}^{\infty} k^{-2}\right)$. Standard calculations (cf. $[9,14])$ prove the lemma. For more details cf. [35]

Lemma 21: Using $\mathbf{L}=\{k \in \mathbf{Z}: k \geq N+1\} \cup\{|N-p|\}$ we get for $n$ even the equation

$$
\begin{aligned}
\mathcal{W}(s)= & 2 c_{W} \sum_{k \in \mathrm{L}}\left(\frac{P\left(-\alpha_{1}^{2}\right)}{\alpha_{1}+k}-\sum_{j=2}^{m} \frac{P\left(-\alpha_{j}^{2}\right)}{\alpha_{j}+k} \frac{\mathcal{P}\left(j, \alpha_{1}\right)}{\mathcal{P}\left(j, \alpha_{j}\right)}\right) \\
& +c_{W} \sum_{\substack{k=-N \\
k \neq N \neq|N-p|}}^{N}\left(\frac{P\left(-\alpha_{1}^{2}\right)}{\alpha_{1}+k}-\sum_{j=2}^{m} \frac{P\left(-\alpha_{j}^{2}\right)}{\alpha_{j}+k} \frac{\mathcal{P}\left(j, \alpha_{1}\right)}{\mathcal{P}\left(j, \alpha_{j}\right)}\right) .
\end{aligned}
$$


Proof: Lemma 21 follows by straightforward calculations starting with (76) using

$$
\pi \tan (\pi \alpha)=-\frac{1}{\alpha-\frac{1}{2}}-\sum_{0 \neq k \in Z}\left(\frac{1}{\alpha-\frac{1}{2}-k}+\frac{1}{k}\right)
$$

and

$$
-P\left(-\alpha_{1}^{2}\right)+\sum_{j=2}^{m} P\left(-\alpha_{j}^{2}\right) \frac{\mathcal{P}\left(j, \alpha_{1}\right)}{\mathcal{P}\left(j, \alpha_{j}\right)}=0
$$

Next we consider (73) for $n$ odd. Then we get

$$
\mathcal{W}(s)=-c_{W} \int_{-\infty}^{+\infty} \tilde{h}(r) d r
$$

with

$$
c_{W}=\frac{\left(\begin{array}{c}
n-1 \\
p
\end{array}\right)(4 \pi)^{-n / 2}}{\Gamma(n / 2)} \operatorname{vol} V, \tilde{h}(r)=\tilde{P}\left(r^{2}\right) \frac{\mathcal{Q}(1, s-N)}{\mathcal{R}(r)}, \tilde{P}(s)=\prod_{\substack{n=0 \\
u \neq i N-p \mid}}^{N}\left(s+u^{2}\right)
$$

The polynomial $\tilde{P}$ is of degree $N=m-1$. Applying (77), we conclude

$$
\tilde{h}(r)=\frac{\tilde{P}\left(-\alpha_{1}^{2}\right)}{r^{2}+\alpha_{1}^{2}}-\sum_{j=2}^{m} \frac{\tilde{P}\left(-\alpha_{j}^{2}\right)}{r^{2}+\alpha_{j}^{2}} \frac{\mathcal{P}\left(j, \alpha_{1}\right)}{\mathcal{P}\left(j, \alpha_{j}\right)} .
$$

Using

$$
\int_{-\infty}^{+\infty} \frac{1}{r^{2}+\alpha^{2}} d r=\frac{\pi}{\alpha}
$$

it follows

$$
\frac{W(s)}{c_{W}}=-\tilde{P}\left(-\alpha_{1}^{2}\right) \frac{\pi}{\alpha_{1}}-\sum_{j=2}^{m} \tilde{P}\left(-\alpha_{j}^{2}\right) \frac{\mathcal{P}\left(j, \alpha_{1}\right)}{\mathcal{P}\left(j, \alpha_{j}\right)} \frac{\pi}{\alpha_{j}} .
$$

Thereby we have proved

Lemma 22: For $n$ odd the function

$$
(s-N) \mathcal{W}(s)=\pi c_{W}\left(-\tilde{P}\left(-(s-N)^{2}\right)+\sum_{j=2}^{m} \cdot \tilde{P}\left(-\alpha_{j}^{2}\right) \frac{\mathcal{P}(j, s-N)}{\mathcal{P}\left(j, \alpha_{j}\right)} \frac{s-N}{\alpha_{j}}\right)
$$

is holomorphic in the whole complex plane.

From Proposition 18 and the definitions (72) and (73) it follows

$$
\Psi_{p}(s)=\sum_{j=2}^{m} \frac{\mathcal{P}(j, s-N)}{\mathcal{P}\left(j, \alpha_{j}\right)} \frac{s-N}{\alpha_{j}} \Psi_{p}\left(\alpha_{j}+N\right)+\kappa_{p} \mathcal{A}(s)+2 \kappa_{p}(s-N) \mathcal{W}(s) .
$$


Proposition 23: The logarithmic derivative $\Psi_{p}$ of the Selberg zeta function $Z_{p}$ has a meromorphic continuation to the whole complex plane. The function $\Psi_{p}$ has holomorphic behaviour with the exception of the following simple poles with integer residues:

\begin{tabular}{|r|l|l|}
\hline no. & \multicolumn{1}{|c|}{ point } & \multicolumn{1}{c|}{ residue } \\
\hline \hline \multirow{3}{*}{ (i) } & \multirow{3}{*}{$s=N-|N-p|$} & $d_{p}^{*}(0)$ for $n$ odd and $p \neq N$ \\
\cline { 3 - 3 } & & $2 d_{p}^{*}(0)$ for $p=N$ \\
\cline { 2 - 3 } & & $\kappa_{p} d_{p}^{*}(0)+(-1)^{n / 2} 2|N-p| \chi(V) P\left(-(N-p)^{2}\right)$ for $n$ even \\
\hline (ii) & $s=N+|N-p|$ & $\kappa_{p} d_{p}^{*}(0)$ for $p \neq N$ \\
\hline (iii) & $s=N$ & $2 \kappa_{p} d_{p}^{*}\left((N-p)^{2}\right)$ \\
\hline (iv) & $s^{+}(\mu)=N+i r_{p}(\mu)$ & \multirow{2}{*}{$\kappa_{p} d_{p}^{*}(\mu)$ for $\mu \neq 0, \mu \neq(N-p)^{2}$} \\
\cline { 2 - 3 } & $s^{-}(\mu)=N-i r_{p}(\mu)$ & $(-1)^{n / 2} 2(N+k) \chi(V) P\left(-(N+k)^{2}\right)$ for $n$ even \\
\hline (v) & $s=-k(k \in N)$ & $(k=N)$ \\
\hline
\end{tabular}

Proof: The proposition is an immediate consequence of (22), (75), (86), Lemma 19 and Lemma 20. We have chosen $\kappa_{p}=\Gamma(p+1) \Gamma(n-p)$ for $n$ even in order to guarantee that $2 \kappa_{p} c_{W}$ is an integer. We remark that $\kappa_{p}$ is in general not the smallest number with that property

Let $M_{p}$ be the number of poles described above, which are real numbers. If we order them by their value, we get $s_{1}, \ldots, s_{M_{p}}$. Gangolli [9] called the poles of type (i) - (iv) spectral poles and those of type (v) topological poles. The topological poles are determined by the Euler-Poincare characteristic $\chi(V)$ and the numbers $p$ and $n$.

As a concequece of Proposition 23 there exists a meromorphic function $Z_{p}(s)$ with $Z_{p}^{\prime}(s) / Z_{p}(s)=\Psi_{p}(s)$, which is uniquely determined up to a multiplicative constant. But this constant is determined by our definition (63). So we have found an analytic continuation of $Z_{p}(s)$ and we get

Theorem 24: The Selberg zeta function. $Z_{p}(s)$ defined by (69) has a meromorphic continuation to the whole complex plane. We define $\nu_{p}=\kappa_{p} d_{p}^{*}(0)$,

$$
\nu_{p}^{*}= \begin{cases}d_{p}^{*}(0) & \text { for } n \text { odd and } p \neq N \\ 2 d_{p}^{*}(0) & \text { for } p=N \\ \kappa_{p} d_{p}^{*}(0)+(-1)^{n / 2} 2|N-p| \chi(V) P\left(-(N-p)^{2}\right) & \text { for } n \text { even }\end{cases}
$$

and $\nu_{p}^{k}=(-1)^{n / 2} 2(N+k) \chi(V) P\left(-(N+k)^{2}\right)$ for $n$ even. The function $Z_{p}(s)$ has holomorphic behaviour with the exception of the following poles and we also state the zeros: 


\begin{tabular}{|r|l|c|}
\hline no. & \multicolumn{1}{|c|}{ pole at point } & multiplicity \\
\hline \hline (i) & $s=N-|N-p|$ for $\nu_{p}^{*}<0$ & $\left|\nu_{p}^{*}\right|$ \\
\hline (ii) & $s=N+|N-p|$ for $\nu_{p}<0$ and $p \neq N$ & $\left|\nu_{p}\right|$ \\
\hline (iii) & $s=-k(k \in N)$ for $n \equiv 0 \bmod 4$ & $\nu_{p}^{k}$ \\
\hline \hline & zero at point & multiplicity \\
\hline \hline (iv) & $s=N-|N-p|$ for $\nu_{p}^{*}>0$ & $\nu_{p}^{*}$ \\
\hline (v) & $s=N+|N-p|$ for $\nu_{p}>0$ and $p \neq N$ & $\nu_{p}$ \\
\hline (vi) & $s=N \quad$ for $(N-p)^{2} \in S_{p}, N \neq p$ & $2 \kappa_{p} d_{p}^{*}\left((N-p)^{2}\right)$ \\
\hline (vii) & $s^{+}(\mu)=N+i r_{p}$ for $\mu \in S_{p}, \mu \neq 0,(N-p)^{2}$ & $\kappa_{p} d_{p}^{*}(\mu)$ \\
\hline (viii) & $s^{-}(\mu)=N-i r_{p}$ for $\mu \in S_{p}, \mu \neq 0,(N-p)^{2}$ & $\kappa_{p} d_{p}^{*}(\mu)$ \\
\hline (ix) & $s=-k(k \in N)$ for $n \equiv 2 \bmod 4$ & $\nu_{p}^{k}$ \\
\hline
\end{tabular}

It follows that the zeros of $Z_{p}(s)$ are in the intervall $[N-|N-p|, N+|N-p|]$, at the points $s=-k,(k \in \mathrm{N})$ or at the line $\operatorname{Re} s=N$. So we can say that the Selberg zeta function $Z_{p}(s)$ satisfies a modified Riemann hypothesis.

In order to get estimations for the Selberg zeta function we will frequently use a functional equation for $Z_{p}(s)$. We define

$$
\Phi_{p}(s)= \begin{cases}(-1)^{n / 2} 2 s \chi(V) P\left(-s^{2}\right) \pi \tan (\pi s) & \text { for } n \text { even } \\
-\left(\begin{array}{c}
n-1 \\
p
\end{array}\right)(4 \pi)^{-(n-2) / 2} \tilde{P}\left(-s^{2}\right) / \Gamma(n / 2) \text { vol } V & \text { for } n \text { odd }\end{cases}
$$

Proposition 25: The logarithmic derivative $\Psi_{p}$ of the Selberg zeta function $Z_{p}$ satisfies the functional equation $\Psi_{p}(s)+\Psi_{p}(2 N-s)=\Phi_{p}(s-N)$.

Proof: From (66) and (72) it follows $\mathcal{A}(s)+\mathcal{A}(2 N-s)=0$. Using (86) we get $\Psi_{p}(s)+\Psi_{p}(2 N-s)=2 \kappa_{p}(s-N)(\mathcal{W}(s)-\mathcal{W}(2 N-s))$. By (76) we obtain for $n$ even $\mathcal{W}(s)-\mathcal{W}(2 N-s)=-2 c_{W} P\left(-(s-N)^{2}\right) \pi \tan (\pi(s-N))$. In view of Lemma 22 we get for $n$ odd the equation $(s-N)(W(s)-W(2 N-s))=-2 \pi c_{W} \tilde{P}\left(-(s-N)^{2}\right)$. Using (75), (81) and the definition (87) the proposition follows at once

Further on, the definition (87) implies

Lemma 26: The function $\Phi_{p}$ is meromorphic in the whole complex plane. It satisfies $\Phi_{p}(s)=\Phi_{p}(-s)$. For $n$ even the function $\Phi_{p}(s)$ is holomorphic with the exception of the simple poles $s= \pm(N-p), s= \pm(N+k)(k \in \mathrm{N})$ with integer residues. The residues at the points $s= \pm(N-p)$ are negative for $n \equiv 2 \bmod 4$. The residues at the points $s= \pm(N-p)$ are positive for $n+2 p \equiv 2 \bmod 4$ for $p<N$ as well as for $n+2 p \equiv 0 \bmod 4$ for $p>N$, in the other cases they are negative. The residues at the points $s= \pm(N+k)$ are negative for $n \equiv 2 \bmod 4$ and positive for $n \equiv 0 \bmod 4$. For $n$ odd the function $\Phi_{p}(s)$ is holomorphic in the whole complex plane.

From Lemma 26 it follows that there exists a meromorphic function $B(s)$ with

$$
\Phi_{p}(s)=\frac{\mathcal{B}_{p}^{\prime}(s)}{\mathcal{B}_{p}(s)} \text { and } \mathcal{B}_{p}(0)=1
$$


As a consequence of Proposition 25 we get

Proposition 27: The Selberg zeta function $Z_{p}$ satisfies the functional equation $Z_{p}(2 N-s)=\mathcal{B}_{p}(N-s) Z_{p}(s)$.

Definition 28: We define the open domain

$$
\begin{aligned}
\mathbf{D}_{p}= & \{s \in \mathbf{C}: \operatorname{Res}>0\} \\
& \backslash \bigcup_{\substack{\mu \in S_{p}^{f} \\
\mu>(N-p)^{2}}}\left(r_{p}(\mu) i, N+r_{p}(\mu) i\right] \\
& \backslash \bigcup_{\substack{\mu \in S_{p}^{f} \\
\mu>(N-p)^{2}}}\left(-r_{p}(\mu) i, N-r_{p}(\mu) i\right] \backslash(0, N+|N-p|] .
\end{aligned}
$$

The domain $\mathbf{D}_{p}$ is simply connected. We have $Z_{p}(s) \neq 0$ for $s \in \mathbf{D}_{p}$. As a consequence of (63) we get

$$
\ln Z_{p}(s)=\kappa_{p} \sum_{\substack{\omega \in \mathbb{N} \\(\omega)=1}} \sum_{k=1}^{q} \sum_{m \in M} \ln \left(1-\epsilon_{p, k}(\omega) \beta^{m}(\omega) e^{-l(\omega)(s+|m|)}\right)
$$

for Res $>2 N$. This describes the function $\ln Z_{p}(s)$ in terms of the length spectrum. The analytic continuation to $\mathbf{D}_{p}$ is meromorphic because of the fact that $\mathbf{D}_{p}$ is simply conected and $Z_{p}(s)$ has no poles and zeros in that domain. Further on we get by analytic continuation

$$
Z_{p}(\bar{s})=\overline{Z_{p}(s)}, \quad \Psi_{p}(\bar{s})=\overline{\Psi_{p}(s)} .
$$

\section{An estimation for the Selberg zeta function based on the Weyl estimation for the eigenvalue spectrum}

We will apply standard methods of prime number theory and function theory. Following Hejhal [14] and Ingham [16] we will omit those parts of the proofs which are straigtforward generalizations, for a detailed discussion cf. Schuster [35]. The main result of this section will be

Theorem 29: For all points $s \in \mathbf{C}$, which have a distance not smaller than $1 / 2$ to the poles of the Selberg zeta function $Z_{p}(s)$, we can state $\left|Z_{p}(s)\right|=\exp \left(O\left(|s|^{n}\right)\right)$.

We remark that in the classical case $n=2, p=0$ describt in [14] there are no poles at all. For $R e s>2 N+\epsilon(0<\epsilon<1)$ Theorem 29 is a simple conclusion of Lemma 14. Next we will analyze the case $\operatorname{Re} s \leq \epsilon$, then it remains to discuss the case $-\epsilon \leq R e s \leq 2 N+\epsilon$. For $n \equiv 2 \bmod 4$ we define

$$
\mathbf{D}_{p}^{\#}=\left\{s \in \mathbf{C}:|s \pm(N-p)|>\frac{1}{4},|s \pm(N+k)|>\frac{1}{4}(k \in \mathbf{N})\right\} \text {. }
$$


For $n \equiv 0(\bmod 4)$ we define

$$
\mathrm{D}_{p}^{\#}=\left\{s \in \mathrm{C}:|s \pm(N-p)|>\frac{1}{4}\right\} .
$$

If $n$ is odd we put $D_{p}^{\#}=C$. We get

Lemma 30: For Res $\leq-\epsilon$ and $s-N \in \mathrm{D}_{p}^{\#}$, we have $\left|Z_{p}(s)\right|=\exp \left(O\left(|s|^{n}\right)\right)$.

Proof: Using $B(s)=B(i) \exp \left(\int_{i}^{s} \Phi_{p}(v) d v\right)$, where the integration shall be taken along the polygonial connecting the points $i, i T$ and $s=\sigma^{*}+i T$ for $I m s \geq 1$ and the points $i, R e s+i$ and $s$ for $0 \leq I m s<1$, a straightforward generalization of [14, pp. 75 - 76] applying the maximum modulus principle gives $\left|B_{p}(s)\right|=\exp \left(O\left(|s|^{n}\right)\right)$ for $s \in \mathbf{D}_{p}^{*}$. The functional equation described in Proposition 27 and Lemma 14/(iii) complete the proof

Following $[14,16]$ we use the concept of "good" numbers. For that we define

$$
x_{k}=\sum_{\substack{\mu \in S_{p} \\ k-2 \leq r p(\mu)<k+2}} d_{p}^{\delta}(\mu)
$$

for $k \in \mathbf{N}, k>2$. The equation (1) implies

$$
\sum_{k=L}^{2 L} x_{k} \sim 4\left(2^{n}-1\right) n_{p} L^{n} . \quad \text { for } L \in \mathrm{N}, L \rightarrow \infty
$$

with $n_{p}$ given by (2). We put $\bar{n}_{p}=4\left(2^{n}-1\right) n_{p}$. The number $k \in N$ is said to be "good" if $x_{k} \leq \bar{n}_{p} k^{n-1}$ is satisfied. By a trivial generalization of [14, Proposition 4.21] we see that for a fixed $\delta>0$ there exists a sufficiently large number $L \in \mathbf{N}$ such that the interval $[L, L(1+\delta)]$ contains "good" values.

But a straightforward generalization of the concept of "admissible" numbers used in [14] would not be strong enough for the following considerations. Our definition will be different also for the classical case $n=2, p=0$. with

Proposition 31: Let $k$ be a "good" number. Then there exists $T \in[k-1, k+1]$

$$
\sum_{T-1 \leq T_{p}(\mu) \leq T+1} \frac{d_{p}^{*}(\mu)}{\left|T-r_{p}(\mu)\right|}=O\left(T^{n-1} \ln T\right) .
$$

Such a number $T$ shall be called "admissible".

For the proof we need the following

Lemma 32: There shall be given real numbers $r_{i}(i=1, \ldots, E)$ with $-2 \leq r_{1} \leq$ $r_{2} \leq \ldots \leq r_{E} \leq 2$. Then there exists a number $r_{.} \in[-1,1]$ with

$$
\sum_{i=1}^{E} \frac{1}{\left|r_{i}-r_{*}\right|}=O(E \ln E) \text {. }
$$

Thereby the $O$-term for $E \rightarrow \infty$ is independent of the position of the points $r_{i}$. 
Proof: We devide the interval $[-2,2)$ into $10 E$ subintervals $I_{i}=\left[-2+(i-1) k_{0},-2+\right.$ $\left.i k_{0}\right)$ with $k_{0}=\frac{2}{s E}, i=1, \ldots, 10 E$. Let $I^{\prime}$ be the union of those intervals $I_{i}$ in which one of the given $r_{j}$ is situated. Let $I^{\prime \prime}$ be the union of intervals which are already included in $I^{\prime}$ or which have neighbouring intervals (with respect to the intervals introduced above) in $I^{\prime}$. We denote by $I^{*}$ the union of the intervals $I_{i}$ which are contained in $[-1,1]$ but which are not included in $I^{\prime \prime}$. By help of the usual Lebesgue measure $\mu$ we get $\mu\left(I^{\prime \prime}\right) \leq 6 / 5$ and $\mu\left(I^{*}\right) \geq 4 / 5$. We denote the midpoint of the interval $I_{j}$ in which the point $r_{i}$ is contained by $\bar{r}_{i}$. It follows $\left|r_{i}-\bar{r}_{i}\right| \leq k_{0} / 2$. For $r \in I^{*}$ and for all $r_{i}, \bar{r}_{i}$ we have $\left|r-r_{i}\right| \geq k_{0},\left|r-\bar{r}_{i}\right| \geq k_{0}$. Further on, we get either $r_{i}>r, \bar{r}_{i}>r$ or $r_{i}<r, \bar{r}_{i}<r$. Suppose $r_{i}>r, \bar{r}_{i}>r$. From $\bar{r}_{i}-r_{i} \leq k_{0} / 2<k_{0}<r_{i}-r$ there follows $\frac{1}{\left|r_{i}-r\right|}<2 \frac{1}{\left|\bar{F}_{i}-r\right|}$. The same equation follows for the other case $r_{i}>r, \bar{r}_{i}>r$ using $r_{i}-\bar{r}_{i} \leq k_{0} / 2<k_{0} \leq r-r_{i}$. We get as an immediate consequence

$$
\sum_{i=1}^{E} \frac{1}{\left|r_{i}-r\right|}<2 \sum_{i=1}^{E} \frac{1}{\left|\bar{r}_{i}-r\right|} \quad \text { for } r \in I^{*} .
$$

We denote by $s_{i}^{*}\left(i=1, \ldots, E^{*}\right)$ the midpoints of those intervals $I_{j}(j=1, \ldots, E)$ for which we have $I_{j} \subset I^{*}$ with $s_{1}^{*}<s_{2}^{*}<\ldots<s_{E}^{*}$. The inequality $\mu\left(I^{*}\right) \geq 4 / 5$ implies $E^{*} \geq 2 E$. We obtain

$$
\sum_{j=1}^{E^{*}} \frac{1}{\left|\bar{r}_{i}-s_{j}^{*}\right|} \leq \frac{2}{k_{0}}\left(\frac{1}{2}+\frac{1}{3}+\frac{1}{4}+\ldots+\frac{1}{10 E}\right) \leq \frac{2}{k_{0}} \ln (10 E)
$$

for $i=1,2,3, \ldots, E$. We deduce that

$$
\sum_{i=1}^{E} \sum_{j=1}^{E^{\bullet}} \frac{i}{\left|\bar{r}_{i}-s_{j}^{*}\right|} \leq \frac{2}{k_{0}} E \ln (10 E)=5 E^{2} \ln (10 E) \text {. }
$$

It follows

$$
\min _{j=1, \ldots, E} \cdot \sum_{i=1}^{E} \frac{1}{\left|\bar{r}_{i}-s_{j}^{*}\right|} \leq \frac{5 E^{2}}{E^{*}} \ln (10 E) .
$$

The minimum shall be reached for $r_{*}=s_{j}^{*}$. Using $\frac{1}{E^{*}} \leq \frac{1}{2 E}$ we get

$$
\sum_{i=1}^{E} \frac{1}{\left|\bar{r}_{i}-r_{\cdot}\right|} \leq \frac{5}{2} E \ln (10 E) \text {. }
$$

Since (92) we find that

$$
\sum_{i=1}^{E} \frac{1}{\left|r_{i}-r_{+}\right|} \leq 5 E \ln (10 E)=O(E \ln E) .
$$

Thereby the lemma is proved

Proof of Proposition 31: By the definition of a "good" number, there are at most $E=\bar{n}_{p} k^{n-1}$ values $r_{p}(\mu)$ (counted with multiplicity $d_{p}^{\delta}(\mu)$ ) which are contained in the interval $[k-2, k+2]$. Applying Lemma 32 there exists $r_{\bullet}=T$ with $T \in[k-1, k+1]$ and

$$
\sum_{k-2 \leq r_{p}(\mu) \leq k+2} \frac{d_{p}^{*}(\mu)}{\left|T-r_{p}(\mu)\right|}=O\left(k^{n-1} \ln k\right)=O\left(T^{n-1} \ln T\right) .
$$


This proves Lemma 31

As mentioned above, we need estimates for the Selberg zeta function $Z_{p}(s)$ for $-\epsilon \leq$ $R e s \leq 2 N+\epsilon$ in order to prove Theorem 29. We can state

Lemma 33: Using $s=N+\sigma+i T$ we suppose $-\frac{1}{2}-N \leq \sigma \leq N+1, T \geq 2$ and that $T$ is "admissible". Then we have

(i) $\left|\Psi_{p}(s)\right|=O\left(|T|^{n}\right)$

(ii) $\left|Z_{p}(s)\right|=\exp \left(O\left(|T|^{n}\right)\right)$. we get

Proof: We start with the case of $n$ even. As a consequence of (72), (79) and (86)

$$
\begin{aligned}
\frac{\Psi_{p}(s)}{s-N}= & \sum_{j=2}^{m} \frac{\mathcal{P}(j, s-N)}{\mathcal{P}\left(j, \alpha_{j}\right)} \frac{\Psi_{p}\left(\alpha_{j}+N\right)}{\alpha_{j}}+2 \kappa_{p} \sum_{\mu \in S_{p}} d_{p}^{F}(\mu) \frac{\mathcal{Q}(1, s-N)}{\mathcal{R}\left(r_{p}(\mu)\right)} \\
& +4 c_{W} \kappa_{p} \sum_{k \in L}\left(\frac{P\left(-\alpha_{1}^{2}\right)}{\alpha_{1}+k}-\sum_{j=2}^{m} \frac{P\left(-\alpha_{j}^{2}\right)}{\alpha_{j}+k} \frac{\mathcal{P}\left(j, \alpha_{1}\right)}{\mathcal{P}\left(j, \alpha_{j}\right)}\right) \\
& +2 c_{\mathcal{W}} \kappa_{p} \sum_{\substack{k \in=N \\
k \neq \pm|N-p|}}^{N}\left(\frac{P\left(-\alpha_{1}^{2}\right)}{\alpha_{1}+k}-\sum_{j=2}^{m} \frac{P\left(-\alpha_{j}^{2}\right)}{\alpha_{j}+k} \frac{\mathcal{P}\left(j, \alpha_{1}\right)}{\mathcal{P}\left(j, \alpha_{j}\right)}\right)
\end{aligned}
$$

By Proposition 23 the poles of $\frac{\Psi_{p}(0)}{-N}$ for the supposed domain of $s$ are at $T= \pm r_{p}(\mu), \sigma=0$ for $\mu \in S_{p}, \mu \geq(N-p)^{2}+4=\mu_{p}^{0}$ with $d_{p}^{*}(\mu) \neq 0$. We deduce

$$
\begin{aligned}
\left|\frac{\Psi_{p}(s)}{s-N}\right| \leq & O\left(|T|^{n-2}\right)+2 \kappa_{p} \sum_{\substack{\mu \in S_{p} \\
\mu_{p}^{\circ} \leq \mu}} d_{p}^{*}(\mu)\left|\frac{\mathcal{Q}(1, \sigma+i T)}{\mathcal{R}\left(r_{p}(\mu)\right)}\right| \\
& +4 c_{W} \kappa_{p} \sum_{k \in L}\left|\frac{P\left(-(\sigma+i T)^{2}\right)}{\sigma+i T+k}-\sum_{j=2}^{m} \frac{P\left(-\alpha_{j}^{2}\right)}{\alpha_{j}+k} \frac{\mathcal{P}(j, \sigma+i T)}{\mathcal{P}\left(j, \alpha_{j}\right)}\right| .
\end{aligned}
$$

We break up the sum $\sum_{\mu_{p}^{0} \leq \mu \in S_{p}}$ into contributions

(i) $r_{p}(\mu)<T-1$,

(ii) $T-1 \leq r_{p}(\mu) \leq T+1$,

(iii) $T+1<r_{p}(\mu)<2 T$,

(iv) $2 T \leq r_{p}(\mu)$.

We recall that we suppose that $T$ is "admissible".

Case $r_{p}(\mu)<T-1$ : With constants $c_{1}$ and $c_{1}^{\prime}$ we obtain

$$
\left|\frac{\mathcal{P}(2, \sigma+i T)}{\mathcal{P}+\left(2, r_{p}(\mu)\right)}\right| \leq c_{1} \frac{T^{n-2}}{\left(r_{p}(\mu)\right)^{n-2}}, \quad\left|\frac{1}{\left(r_{p}(\mu)\right)^{2}+\alpha_{2}^{2}} \frac{\mathcal{P}(2, \sigma+i T)}{\mathcal{P}+\left(2, r_{p}(\mu)\right)}\right| \leq c_{1}^{\prime} \frac{T^{n-2}}{\left(r_{p}(\mu)\right)^{n}} .
$$

Using $\left|T-r_{p}(\mu)\right|>1$, we see that

$$
\left|\frac{1}{\left(r_{p}(\mu)\right)^{2}+(\sigma+i T)^{2}}\right|=\left|\frac{1}{\left(r_{p}(\mu)+i \sigma-T\right)\left(r_{p}(\mu)-i \sigma+T\right)}\right| \leq \frac{1}{r_{p}(\mu)} .
$$


The spectral estimate (24) implies

$$
\sum_{\substack{\mu \in S_{p} \\ r_{p}(\mu)<T-1}} \frac{d_{p}^{*}(\mu)}{\left(r_{p}(\mu)\right)^{n-1}}=O(T), \quad \sum_{\substack{\mu \in S_{p} \\ r_{p}(\mu)<T-1}} \frac{d_{p}^{*}(\mu)}{\left(r_{p}(\mu)\right)^{n}}=O(\ln T) .
$$

We obtain

$$
\begin{aligned}
& \sum_{\substack{\mu \in S_{p} \\
\mu, 0<\mu, r_{p}(\mu)<T-1}} d_{p}^{*}(\mu)\left|\frac{\mathcal{Q}(1, \sigma+i T)}{\mathcal{R}\left(r_{p}(\mu)\right)}\right| \\
& \leq \sum_{\substack{\mu \in S_{p} \\
\mu j<\mu, r_{p}(\mu)<T-1}} d_{p}^{*}(\mu)\left|\left(\frac{1}{\left(r_{p}(\mu)\right)^{2}+(\sigma+i T)^{2}}-\frac{1}{\left(r_{p}(\mu)\right)^{2}+\alpha_{2}^{2}}\right) \frac{\mathcal{P}(2, \sigma+i T)}{\mathcal{P}+\left(2, r_{p}(\mu)\right)}\right| \\
& =O\left(T^{n-1}\right) .
\end{aligned}
$$

Case $T-1 \leq r_{p}(\mu) \leq T+1$ : Here we have to use that $T$ is "admissiblen. We get

$$
\begin{aligned}
\sum_{\substack{\mu \in S_{p} \\
T-1 \leq r_{p}(\mu) \leq T+1}} d_{p}^{*}(\mu)\left|\frac{\mathcal{Q}(1, \sigma+i T)}{\mathcal{R}\left(r_{p}(\mu)\right)}\right| & =\left(\sum_{\substack{\mu \in s_{p} \\
T-1 \leq p}} d_{p}^{*}(\mu) \frac{1}{\left|r_{p}(\mu)-T\right| \leq T+1}\right) O\left(\frac{1}{T}\right) \\
& =O\left(T^{n-2} \ln T\right) \\
& =O\left(T^{n-1}\right) .
\end{aligned}
$$

Case $T+1<r_{p}(\mu)<2 T$ : We get the same result as for the case $r_{p}(\mu)<T-1$ because of the fact that we have $\left|T-r_{p}(\mu)\right|>1$ again.

Case $2 T<r_{p}(\mu)$ : We clearly obtain

$$
\left|\frac{1}{\left(r_{p}(\mu)\right)^{2}+(\sigma+i T)^{2}}\right|=\frac{1}{\left|r_{p}(\mu)+i \sigma-T\right|\left|r_{p}(\mu)-i \sigma+T\right|} \leq \frac{2}{\left(r_{p}(\mu)\right)^{2}} .
$$

The estimation (25) implies

$$
\sum_{\substack{\mu \in S_{p} \\ r_{p}(\mu) \geq 2 r}} d_{p}^{*}(\mu) \frac{1}{\left(r_{p}(\mu)\right)^{n+2}}=O\left(T^{-2}\right)
$$

and thereby

$$
\sum_{\substack{\mu \in S_{p} \\ r p(\mu) \geq 2 T}} d_{p}^{*}(\mu)\left|\frac{\mathcal{Q}(1, \sigma+i T)}{\mathcal{R}\left(r_{p}(\mu)\right)}\right|=O\left(T^{n-2}\right)
$$

Summarizing the 4 cases considered above, we get

$$
2 \kappa_{p} \sum_{\substack{\mu \in S_{p} \\ \mu \neq 0<\mu}} d_{p}^{*}(\mu)\left|\frac{\mathcal{Q}(1, \sigma+i T)}{\mathcal{R}\left(r_{p}(\mu)\right)}\right|=O\left(T^{n-1}\right)
$$


Next we consider the sum $\sum_{k \in L}$ in (93). We break up the sum into two parts with $k<2 T$ and $k \geq 2 T$. Using $T>2$ we get

$$
\begin{aligned}
4 c_{W} \sum_{k=N+1}^{[2 T]} & \left(\frac{P\left(-(s-N)^{2}\right)}{s-N+k}-\sum_{j=2}^{m} \frac{P\left(-\alpha_{j}^{2}\right)}{\alpha_{j}+k} \frac{\mathcal{P}\left(j, \alpha_{1}\right)}{\mathcal{P}\left(j, \alpha_{j}\right)}\right) \\
& +4 c_{W}\left(\frac{P\left(-(s-N)^{2}\right)}{s-N+|p-N|}-\sum_{j=2}^{m} \frac{P\left(-\alpha_{j}^{2}\right)}{\alpha_{j}+|p-N|} \frac{\mathcal{P}\left(j, \alpha_{1}\right)}{\mathcal{P}\left(j, \alpha_{j}\right)}\right) \\
= & O\left(T^{n-2}\right) \sum_{k=N+1}^{[2 T]}\left(\frac{1}{T}+\frac{1}{k+1}\right)+O\left(T^{n-2}\right)\left(\frac{1}{T}+\frac{1}{|p-N|+1}\right) \\
= & O\left(T^{n-2} \ln T\right)+O\left(T^{n-2}\right) \\
= & O\left(T^{n-1}\right) .
\end{aligned}
$$

It is easily seen that

$$
P\left(-(s-N)^{2}\right)-\sum_{j=2}^{m} P\left(-\alpha_{j}^{2}\right) \frac{\mathcal{P}\left(j, \alpha_{1}\right)}{\mathcal{P}\left(j, \alpha_{j}\right)}=0 .
$$

It follows that

$$
\begin{gathered}
4 c_{W} \sum_{k=\{2 T+1]}^{\infty}\left(\frac{P\left(-(s-N)^{2}\right)}{s-N+k}-\sum_{j=2}^{m} \frac{P\left(-\alpha_{j}^{2}\right)}{\alpha_{j}+k} \frac{\mathcal{P}\left(j, \alpha_{1}\right)}{\mathcal{P}\left(j, \alpha_{j}\right)}\right) \\
=\sum_{k=[2 T+1]}^{\infty}\left(\frac{O\left(k^{m-2}\right) T^{n-2}}{(s-N+k) \prod_{j=2}^{m}\left(\alpha_{j}+k\right)}\right) \\
=O\left(T^{n-2}\right) .
\end{gathered}
$$

This comletes the proof of the first part of Lemma 33. In order to prove (ii) we integrate $\Psi_{p}(s)=\frac{Z_{p}^{\prime}(s)}{Z_{p}(s)}$ along the straight line from $2 N+1+i T$ to $\sigma+N+i T$. For $n$ odd we get a similar estimation -

Proof of Theorem 29: The theorem is an imediate consequence of (88), Lemma 30 , Lemma 33 and the maximum modulus principle

\section{Spectral estimations based on estimations for the zeta function}

In this section we prove a weaker version of Theorem $B$. We need this result in order to get estimations for the logarithmic derivative of the Selberg zeta function (cf. Section 10) as a tool for the proof of Theorem B (cf. Section 11). As we remarked in the Introduction (Part I), this weaker version is well known in the literature. The proof is reached easily generalizing [14]. We mainly want to point out, which tools are necessary for our approach, and we give those details which we will need later on. For more details cf. Schuster [35]. 
Proposition 34: The error term $\mathcal{R}_{p}(T)$ defined by

$$
\sum_{\substack{\mu \in S_{p} \\
\mu<T^{2}+(p-N)^{2}}} d_{p}^{\delta}(\mu)=\frac{\left(\begin{array}{c}
n-1 \\
p
\end{array}\right) \text { vol } V}{(4 \pi)^{n / 2} \Gamma\left(\frac{n+2}{2}\right)} T^{n}+\mathcal{R}_{p}(T)
$$

satisfies $\left|\mathcal{R}_{p}(T)\right|=O\left(T^{n-1}\right)$.

We will prove Proposition 34 after Proposition 38. For $s \in \mathbf{D}_{p}$ (cf. Definition 28) we define

$$
\arg Z_{p}(s)=I m \ln Z_{p}(s)
$$

The following proposition states a connection between $\arg Z_{p}(s)$ and the p-eigenvalue spectrum.

Proposition 35: For $T \geq 1, T \notin\left\{r_{p}(\mu): \mu \in S_{p}\right\}$ we obtain

$$
\mathcal{N}_{p}(T)=n_{p} T^{n}+\mathcal{E}_{p}(T)+\mathcal{H}_{p}(T)
$$

with

$$
\begin{aligned}
\mathcal{E}_{p}(T) & = \begin{cases}(-1)^{n / 2} 2 \chi(V) \int_{0}^{T}\left[t P\left(t^{2}\right) \tanh t-t^{n-1}\right] d t+\frac{m_{p}^{*}}{2 \kappa_{p}} & \text { for } n \text { even } \\
\frac{\left(\begin{array}{l}
n-1 \\
p
\end{array}\right) \text { vol } V}{(4 x)^{n / 2} \Gamma\left(\frac{n}{2}\right)} \int_{0}^{T}\left[\tilde{P}\left(t^{2}\right)-t^{n-1}\right] d t+\frac{m_{p}^{*}}{2} & \text { for } n \text { odd, }\end{cases} \\
\mathcal{H}_{p}(T) & =\frac{1}{\pi \kappa_{p}} \arg Z_{p}(N+i T)
\end{aligned}
$$

with an integer $m_{p}^{*}$ depending only on $p$ and the space form $V$.

Proof: Proposition 35 is a generalization of [14, Theorem 7.1]. For the straightforward generalization of the proof one has to use the Cauchy residue theorem, Proposition 23, Lemma 26, Proposition 27 and (88)

An easy computation shows

Lemma 36: We observe that $\mathcal{E}_{p}(T)=O\left(T^{n-2}\right)$.

Proposition 37: For $\sigma^{*} \geq-\frac{1}{2}$ and $T \geq 1$ we get

$$
\left|Z_{p}\left(\sigma^{*}+i T\right)\right| \leq \exp \left(a_{p} T^{n-1}+O\left(T^{n-2}\right)\right)
$$

with

$$
a_{p}= \begin{cases}(-1)^{n / 2} \pi n \chi(V) & \text { for } n \text { even } \\ -\frac{n(n-1) \text { vol } V}{2(4 \pi)^{(n-2) / 2} \Gamma(n / 2)} & \text { for } n \text { odd }\end{cases}
$$

Proof: The related proof of [14] for $n=2, p=0$ as well as the straightforward generalization to our situation is based on the Phragmén-Lindelöf principle (cf. Boas [3, Theorem 1.4.2]) and the functional equation of Proposition 27 . 
Using an estimation of Titchmarsh [26, Lemma 9.4] and Jensens' Theorem (Boas [3, Theorem 1.2.1]), Proposition 37 implies the following proposition (cf. Hejhal [14] for $n=2, p=0)$.

Proposition 38: For $T \geq 1, T \notin\left\{r_{p}(\mu), \mu \in S_{p}\right\}$, we have $\left|\arg Z_{p}(N+i T)\right|=$ $O\left(T^{n-1}\right),\left|\mathcal{H}_{p}(T)\right|=O\left(T^{n-1}\right)$.

Proof of Proposition 34: By virtue of Proposition 35, Lemma 36, Proposition 38 and a usual continuity argument we get the assertion -

\section{Estimations for the logarithmic derivative of the Selberg zeta function \\ using spectral estimations}

As we have already remarked, we want to use Proposition 34 in order to get estimations of $\Psi_{p}(s)$.

Proposition 39: Supposing $s=N+\sigma+i T,-\frac{1}{2}-N \leq \sigma \leq N+1, T \geq 100$, $T \notin\left\{r_{p}(\mu): \mu \in S_{p}\right\}$ we get

$$
\frac{\Psi_{p}(s)}{s-N}=O\left(|T|^{n-2}\right)+2 \kappa_{p} \sum_{\substack{\mu \in s_{p} \\ T-1 \leq r_{p}(\mu) \leq T+1}} d_{p}^{*}(\mu) \frac{\mathcal{Q}(1, s-N)}{\mathcal{R}\left(r_{p}(\mu)\right)}
$$

Proof: We suppose $\alpha_{m}>\alpha_{m-1}>\ldots>\alpha_{2}>n$ with $m=\frac{n}{2}+1$ for $n$ even and $m=\frac{n+1}{2}$ for $n$ odd (as above, cf. Assumption 16). We start with the case of $n$ even. As a consequence of (72), (79) and (86) we get in analogy to (93) (cf. the proof of Lemma 33) the equation

$$
\begin{aligned}
\frac{\Psi_{p}(s)}{s-N}= & O\left(|T|^{n-2}\right)+2 \kappa_{p} \sum_{\substack{\mu \in S_{p} \\
r p(\mu) \geq 0}} d_{p}^{*}(\mu) \frac{\mathcal{Q}(1, s-N)}{\mathcal{R}\left(r_{p}(\mu)\right)} \\
& +4 c_{W} \kappa_{p} \sum_{k \in \mathbf{L}}\left(\frac{P\left(-(\sigma+i T)^{2}\right)}{\sigma+i T+k}-\sum_{j=2}^{m} \frac{P\left(-\alpha_{j}^{2}\right)}{\alpha_{j}+k} \frac{\mathcal{P}(j, \sigma+i T)}{\mathcal{P}\left(j, \alpha_{j}\right)}\right) .
\end{aligned}
$$

Thereby the assumption $r_{p}(\mu)>0$ shall include the assumption $r_{p}(\mu) \in \mathbf{R}$. We break up the sum $\sum_{\substack{\mu \in S_{p} \\ r p(\mu) \geq 0}}$ in the same way as we have done it with the sum $\sum_{\substack{\mu \in S_{p} \\ \mu D}}$ in the proof of Lemma 33.

Case $r_{p}(\mu)<T-1$ : We choose a number $\delta$ with $2 N<\delta<2 N+1$ and $\delta \notin\left\{r_{p}(\mu)\right.$ : $\left.\mu \in S_{p}\right\}$. Then we get

$$
S_{1}=2 \kappa_{p} \sum_{\substack{\mu \in S_{p} \\ r p(\mu)<T-1}} d_{p}^{*}(\mu) \frac{\mathcal{Q}(1, s-N)}{\mathcal{R}\left(r_{p}(\mu)\right)}
$$


We define

$$
\begin{gathered}
=O\left(T^{n-2}\right)+2 \kappa_{p} \int_{\delta}^{T-1} \frac{\mathcal{Q}(1, s-N)}{\mathcal{R}(r)} d \mathcal{N}_{p}(r) \\
=O\left(T^{n-2}\right)+2 \kappa_{p} \int_{\delta}^{T-1}\left(\frac { 1 } { 2 ( \sigma + i T ) } \left[\frac{1}{\sigma+i(T+r)}\right.\right. \\
\left.\left.\quad+\frac{1}{\sigma+i(T-r)}\right]-\frac{1}{r^{2}+\alpha_{2}^{2}}\right) \frac{\mathcal{P}(2, s-N)}{\mathcal{P}+(2, r)} d \mathcal{N}_{p}(r) .
\end{gathered}
$$

It follows

$$
I_{1}=\frac{\kappa_{p}}{\sigma+i T} \int_{\delta}^{T-1} \frac{1}{\sigma+i(T+r)} \frac{\mathcal{P}(2, s-N)}{\mathcal{P}+(2, r)} d \mathcal{N}_{p}(r)
$$

$$
\left|I_{1}\right| \leq \frac{\kappa_{p}}{T}|\mathcal{P}(2, s-N)| \int_{\delta}^{T-1} \frac{1}{(T+r) r^{n-2}} d \mathcal{N}_{p}(r) \leq O\left(T^{n-4}\right) \int_{\delta}^{T-1} \frac{1}{r^{n-2}} d \mathcal{N}_{p}(r) .
$$

The Weyl asymptotic estimate (1) states $\mathcal{N}_{p}(r)=O\left(r^{n}\right)$ and thereby we get by partial integration $\left|I_{1}\right|=O\left(T^{n-2}\right)$. Next we consider

$$
I_{2}=\frac{\kappa_{p}}{\sigma+i T} \int_{\delta}^{T-1} \frac{1}{\sigma+i(T-r)} \frac{\mathcal{P}(2, s-N)}{\mathcal{P}+(2, r)} d \mathcal{N}_{p}(r) .
$$

Now we use Proposition 34. By help of $\mathcal{N}_{p}(T)=n_{p} T^{n}+\mathcal{R}_{p}(T)$ we break up $I_{2}$ into $I_{2}=I_{2}^{\prime}+I_{2}^{\prime \prime}$ with

$$
I_{2}^{\prime}=\frac{\kappa_{p}}{\sigma+i T} \int_{\delta}^{T_{-1}} \frac{1}{\sigma+i(T-r)} \frac{\mathcal{P}(2, s-N)}{\mathcal{P}+(2, r)} d\left(n_{p} r^{n}\right) .
$$

We obtain

$$
\begin{aligned}
I_{2}^{\prime} & =\frac{\kappa_{p}}{\sigma+i T} \mathcal{P}(2, s-N) \int_{\delta}^{T-1} \frac{n n_{p} r^{n-1}}{(\sigma+i(T-r)) \mathcal{P}+(2, r)} d r \\
& =\frac{\kappa_{p}}{\sigma+i T} \mathcal{P}(2, s-N) \int_{\delta}^{T-1} \frac{n n_{p} r \mathcal{P}+(2, r)+P_{n-3}(r)}{(\sigma+i(T-r)) \mathcal{P}+(2, r)} d r \\
& =\frac{\kappa_{p}}{\sigma+i T} \mathcal{P}(2, s-N) n n_{p}\left(\int_{\delta}^{T-1} \frac{r}{\sigma+i(T-r)} d r+O(1)\right)
\end{aligned}
$$

with a polynomial $P_{n-3}(r)$ of degree $n-3$. Further on we use

$$
\begin{aligned}
\int_{\delta}^{T-1} \frac{r}{\sigma+i(T-r)} d r & =-i \int_{\delta}^{T-1} \frac{i(r-T)-\sigma}{\sigma+i(T-r)} d r+i \int_{\delta}^{T-1} \frac{-i T-\sigma}{\sigma+i(T-r)} d r \\
& =i(T-1-\delta)+(i T+\sigma) \int_{\delta}^{T-1} \frac{1}{\sigma i+(r-T)} d r \\
& =i(T-1-\delta)+\left.(i T+\sigma) \ln (\sigma i+r-T)\right|_{\delta} ^{T-1}
\end{aligned}
$$


It follows

$$
\frac{1}{\sigma+i T} \int_{\delta}^{T-1} \frac{r}{\sigma+i(T-r)} d r=-\ln T+O(1)
$$

and thereby

$$
I_{2}^{\prime}=-\kappa_{p} n n_{p} \mathcal{P}(2, s-N) \ln T+O\left(T^{n-2}\right)
$$

Further on we get for

$$
I_{2}^{\prime \prime}=\frac{\kappa_{p}}{\sigma+i T} \int_{\delta}^{T_{-1}} \frac{1}{\sigma+i(T-r)} \frac{\mathcal{P}(2, s-N)}{\mathcal{P}+(2, r)} d \mathcal{R}_{p}(r)
$$

the estimation

$$
\begin{aligned}
\left|I_{2}^{\prime \prime}\right| & \leq \frac{\kappa_{p}}{T}|\mathcal{P}(2, s-N)| \int_{\delta}^{T-1} \frac{1}{(T-r) r^{n-2}} d\left|\mathcal{R}_{p}(r)\right| \\
& \leq \frac{\kappa_{p}}{T}|\mathcal{P}(2, s-N)|\left(\left.\frac{\left|\mathcal{R}_{p}(r)\right|}{(T-r) r^{n-2}}\right|_{\delta} ^{T-1}+\int_{\delta}^{T-1}\left|\mathcal{R}_{p}(r)\right| \frac{\left|(n-1) r^{n-2}-T(n-2) r^{n-3}\right|}{(T-r)^{2} r^{2 n-4}} d r\right) \\
& \leq O\left(T^{n-3}\right)\left(O(T)+\int_{\delta}^{T-1}\left|O\left(r^{n-1}\right)\right| \frac{|(n-1) r-T(n-2)|}{(T-r)^{2} r^{n-1}} d r\right) \\
& \leq O\left(T^{n-3}\right)\left(O(T)+\int_{\delta}^{T-1}|O(1)| \frac{|(n-1) r-T(n-2)|}{(T-r)^{2}} d r\right)
\end{aligned}
$$

and therby $\left|I^{\prime \prime}\right|=O\left(T^{n-2}\right)$. We conclude

$$
I_{2}=-\kappa_{p} n n_{p} \mathcal{P}(2, s-N) \ln T+O\left(T^{n-2}\right) .
$$

For the estimation of $S_{1}$ it remains to.consider

$$
I_{3}=-2 \kappa_{p} \int_{\delta}^{T-1} \frac{\mathcal{P}(2, s-N)}{\mathcal{Q}^{+}(1, r)} d \mathcal{N}_{p}(r)
$$

We find that

$$
I_{3}=-2 \kappa_{p} n n_{p} \mathcal{P}(2, s-N) \int_{\delta}^{T-1} \frac{r^{n-1}}{\mathcal{Q}^{+}(1, r)} d r-2 \kappa_{p} \mathcal{P}(2, s-N) \int_{\delta}^{T_{-1}} \frac{1}{\mathcal{Q}^{+}(1, r)} d \mathcal{R}_{p}(r)
$$

In analogy to the estimation of $I_{2}^{\prime}$ and $I_{2}^{\prime \prime}$ we obtain

$$
I_{3}=-2 \kappa_{p} n n_{p} \mathcal{P}(2, s-N) \ln T T^{n-2}+O\left(T^{n-2}\right) .
$$

An easy calculation shows $\mathcal{P}(2, s-N)=T^{n-2}+O\left(T^{n-3}\right)$, and we immediately obtain

$$
S_{1}=-3 \kappa_{p} n n_{p} \ln T T^{n-2}+\dot{O}\left(T^{n-2}\right) \text {. }
$$


In the case $T-1 \leq r_{p}(\mu) \leq T+1$ we have to do no changes for the assertion of the proof.

Next we consider the case $T+1<r_{p}(\mu)<2 T$. We then have to estimate

$$
S_{3}=2 \kappa_{p} \sum_{\substack{\mu \in S_{p} \\ T+1<r_{p}(\mu)<2 T}} d_{p}^{*}(\mu) \frac{\mathcal{Q}(1, s-N)}{\mathcal{R}\left(r_{p}(\mu)\right)} .
$$

If $r_{p}(\mu)=T+1$ or $r_{p}(\mu)=2 T$, then Proposition 34 implies $d_{p}^{*}(\mu)=O\left(T^{n-1}\right)$. We conclude

$$
S_{3}=O\left(T^{n-2}\right)+2 \kappa_{p} \int_{T+1}^{2 T} \frac{\mathcal{Q}(1, s-N)}{\mathcal{R}\left(r_{p}(\mu)\right)} \mathcal{N}_{p}(r) .
$$

We use the decomposition

$$
\frac{\mathcal{Q}(1, s-N)}{\mathcal{R}\left(r_{p}(\mu)\right)}=\left(\frac{1}{2(\sigma+i T)}\left(\frac{1}{\sigma+i(T+r)}+\frac{1}{\sigma+i(T-r)}\right)-\frac{1}{r^{2}+\alpha_{2}^{2}}\right) \frac{\mathcal{P}(2, s-N)}{\mathcal{P}+(2, r)}
$$

as above. First we consider

$$
J_{1}=\frac{\kappa_{p}}{\sigma+i T} \int_{T+1}^{2 T} \frac{1}{\sigma+i(T+r)} \frac{\mathcal{P}(2, s-N)}{\mathcal{P}+(2, r)} d \dot{\mathcal{N}}_{p}(r)
$$

We get

$$
\left|J_{1}\right| \leq \frac{\kappa_{p}}{T^{2}}|\mathcal{P}(2, s-N)| \int_{T+1}^{2 T} \frac{1}{r^{n-2}} d \mathcal{N}_{p}(r)
$$

and therby $J_{1}=O\left(T^{n-2}\right)$. In the same way we get for

$$
J_{3}=2 \kappa_{p} \int_{T+1}^{2 T} \frac{\mathcal{P}(2, s-N)}{\mathcal{Q}^{+}(1, r)} d \mathcal{N}_{p}(r)
$$

the estimation $J_{3}=O\left(T^{n-2}\right)$. It remains to consider

$$
J_{2}=\frac{\kappa_{p}}{\sigma+i T} \mathcal{P}(2, s-N) \int_{T+1}^{2 T} \frac{1}{\sigma+i(T-r)} \frac{1}{\mathcal{P}+(2, r)} \mathcal{N}_{p}(r)
$$

According to the decomposition $\mathcal{N}_{p}(r)=n_{p} r^{n}+\mathcal{R}_{p}(r)$, we study

$$
J_{2}^{\prime}=\frac{\kappa_{p}}{\sigma+i T} \mathcal{P}(2, s-N) \int_{T+1}^{2 T} \frac{1}{\sigma+i(T-r)} \frac{n n_{p} r^{n-1}}{\mathcal{P}+(2, r)} d r .
$$

We have

$$
\int_{T+1}^{2 T} \frac{1}{\sigma+i(T-r)} \frac{r^{n-1}}{\mathcal{P}+(2, r)} d r
$$




$$
\begin{aligned}
& =\int_{T+1}^{2 T} \frac{1}{\sigma+i(T-r)}\left(r+O\left(\frac{1}{r}\right)\right) d r \\
& =\int_{T+1}^{2 T}\left(\frac{(r-T)+i \sigma}{\sigma+i(T-r)}+\frac{T-i \sigma}{\sigma+i(T-r)}+\frac{O(1 / r)}{\sigma+i(T-r)}\right) d r \\
& =O(T)+(\sigma+i T) \int_{T+1}^{2 T} \frac{1}{\sigma i-T+r} d r \\
& =O(T)+(\sigma+i T) \ln T .
\end{aligned}
$$

It follows $J_{2}^{\prime}=\kappa_{p} n n_{p} T^{n-2} \ln T+O\left(T^{n-2}\right)$. In analogy to the calculations above, we get

$$
J_{2}^{\prime \prime}=\frac{\kappa_{p}}{\sigma+i T} \mathcal{P}(2, s-N) \int_{T+1}^{2 T} \frac{1}{\sigma+i(T-r)} \frac{1}{\mathcal{P}+(2, r)} d \mathcal{R}_{p}(r)=O\left(T^{n-2}\right)
$$

It follows

$$
S_{3}=\kappa_{p} n n_{p} T^{n-2} \ln T+O\left(T^{n-2}\right) .
$$

We recall that for the case $2 T<r_{p}(\mu)$ we have shown in Section 8 the estimation

$$
S_{4}=2 \kappa_{p} \sum_{\substack{\mu \in S_{p} \\ 2 T<r_{p}(\mu)}} d_{p}^{*}(\mu)\left|\frac{\mathcal{Q}(1, \sigma+i T)}{\mathcal{R}\left(r_{p}(\mu)\right)}\right|=O\left(T^{n-2}\right)
$$

We now turn our attention to the estimation of the sum $\sum_{k \in L}$ in the right-hand side of (100) using an argument of the proof of Lemma 33. We define

$$
S_{\mathrm{s}}=4 c_{W} \kappa_{p} \sum_{k \in \mathbf{L}_{T}}\left(\frac{P\left(-(\sigma+i T)^{2}\right)}{\sigma+k+i T}-\sum_{j=2}^{m} \frac{P\left(-\alpha_{j}^{2}\right)}{\alpha_{j}+k} \frac{\mathcal{P}(j, \sigma+i T)}{\mathcal{P}\left(j, \alpha_{j}\right)}\right)
$$

with $\mathbf{L}_{T}=\{k \in \mathbf{L}: k \leq 2 T\}$. We apply

$$
\ln (\alpha+B+1)-\ln (\alpha+A)<\sum_{k=A}^{B} \frac{1}{\alpha+k}<\ln (\alpha+B)-\ln (\alpha+A+1)
$$
with $A, B \in \mathbf{N}, \alpha \in \mathbf{R}, \alpha>0$ and get $\sum_{k \in \mathbf{L}_{T} \frac{1}{\alpha+k}}=\ln T+O(1)$. We immediately see
that

$$
\begin{aligned}
S_{5} & =4 c_{\mathcal{W} \kappa_{p}}\left(-\sum_{j=2}^{m} P\left(-\alpha_{j}^{2}\right) \frac{\mathcal{P}(j, \sigma+i T)}{\mathcal{P}\left(j, \alpha_{j}\right)} \ln T\right)+O\left(T^{n-2}\right) \\
& =-4 c_{W} \kappa_{p} P\left(-(s-N)^{2}\right) \ln T+O\left(T^{n-2}\right) \\
& =-4 c_{\mathcal{W}} \kappa_{p} T^{n-2} \ln T+O\left(T^{n-2}\right) .
\end{aligned}
$$

Equation (97) states

$$
S_{5}=4 c_{W \kappa_{p}} \sum_{k=\{2 T]+1}^{\infty}\left(\frac{P\left(-(\sigma+i T)^{2}\right)}{\sigma+k+i T)}-\sum_{j=2}^{m} \frac{P\left(-\alpha_{j}^{2}\right)}{\alpha_{j}+k} \frac{\mathcal{P}(j, \sigma+i T)}{\mathcal{P}\left(j, \alpha_{j}\right)}\right)=O\left(T^{n-2}\right)
$$


Summarizing (100) - (103) it follows

$$
\frac{\Psi_{p}(s)}{s-N}=O\left(T^{n-2}\right)-2 \kappa_{p} n n_{p} \ln T T^{n-2}-4 c_{W} \kappa_{p} \ln T T^{n-2} .
$$

Using

$$
n_{p}=(-1)^{n / 2} \frac{\left(\begin{array}{c}
n-1 \\
p
\end{array}\right) \chi(V)}{\Gamma(n+1)} \text { and } c_{W}=(-1)^{\frac{n+2}{2}} \frac{\chi(V)}{2 \Gamma(p+1) \Gamma(n-p)}
$$

we get the assertion of the proposition for $n$ even.

Next we consider the case of $n$ odd. Instead of (100) we get

$$
\frac{\Psi_{p}(s)}{s-N}=O\left(|T|^{n-2}\right)+2 \sum_{\substack{\mu \in S_{p} \\ r p(\mu) \geq 0}} d_{p}^{*}(\mu) \frac{\mathcal{Q}(1, s-N)}{\mathcal{R}\left(r_{p}(\mu)\right)}
$$

We use the same decomposition of the sum on the right-hand side as in the case of $n$ even. In contrast to this case we get

$$
\begin{aligned}
I_{2}^{\prime} & =\frac{\mathcal{P}(2, s-N)}{\sigma+i T} \int_{\delta}^{T-1} \frac{n n_{p} r^{n-1}}{(\sigma+i(T-r)) \mathcal{P}+(2, r)} d r \\
& =\frac{\mathcal{P}(2, s-N)}{\sigma+i T} \int_{\delta}^{T-1} \frac{n n_{p} r^{2} \mathcal{P}^{+}(2, r)+P_{n-3}(r)}{(\sigma+i(T-r)) \mathcal{P}+(2, r)} d r
\end{aligned}
$$

with a polynomial $P_{n-3}(r)$ of degree $n-3$ and consequently

$$
I_{2}^{\prime}=\frac{n n_{p} \mathcal{P}(2, s-N)}{\sigma+i T}\left(\int_{\delta}^{T-1} \frac{r^{2}}{\sigma+i(T-r)}+\int_{\delta}^{T-1} \frac{O(1)}{\sigma+i(T-r)} d r\right) .
$$

We apply

$$
\int_{\delta}^{T-1} \frac{r^{2}}{\sigma+i(T-r)} d r=i \int_{\delta}^{T-1} r d r-i(\sigma+i T) \int_{\delta}^{T-1} \frac{r}{\sigma+i(T-r)} d r=O\left(T^{2}\right)
$$

and get $I_{2}^{\prime}=O\left(T^{n-2}\right)$. In the same way we get $I_{3}=O\left(T^{n-2}\right)$. It follows $I_{1}=O\left(T^{n-2}\right)$. A similar calculation gives $S_{3}=O\left(T^{n-2}\right)$. This completes the proof

Proposition 40: For $-\frac{1}{2} \leq R e s \leq n, \quad I m s \geq 100, s=N+\sigma+i T$ and $T \notin$ $\left\{r_{p}(\mu): \mu \in S_{p}\right\}$ we obtain

$$
\Psi_{p}(s)=O\left(T^{n-1}\right)+\kappa_{p} \sum_{\substack{\mu \in S_{p} \\|r p(\mu)-T| \leq 1}} \frac{d_{p}^{*}(\mu)}{s-N-i r_{p}(\mu)}
$$

Proof: Proposition 39 implies

$$
\begin{aligned}
\Psi_{p}(s)= & O\left(T^{n-1}\right)+\kappa_{p} \sum_{\substack{\left|\mu \in s_{p}\\
\right| r_{p}(\mu)-T_{\mid} \leq 1}} d_{p}^{*}(\mu)\left(\left(\frac{1}{s-N-i r_{p}(\mu)}\right.\right. \\
& \left.\left.+\frac{1}{s-N+i r_{p}(\mu)}-\frac{2(s-N)}{r_{p}^{2}(\mu)+\alpha_{2}^{2}}\right) \frac{\mathcal{P}(2, s-N)}{\mathcal{P}+\left(2,, r_{p}(\mu)\right.}\right) .
\end{aligned}
$$


Using $-\frac{1}{2} \leq R e s \leq n$ and $\left|r_{p}(\mu)-T\right| \leq 1$ we conclude

$$
\frac{\mathcal{P}(2, s-N}{\mathcal{P}+(2, r)}=(-1)^{m-2}+O\left(\frac{1}{T^{2}}\right) \text {. }
$$

As a consequence of Proposition 34 we get $\mathcal{N}_{p}(T+2)-\mathcal{N}_{p}(T-2)=O\left(T^{n-1}\right)$ and thereby

$$
\begin{aligned}
\sum_{\substack{\mu \in S_{p} \\
\left|r_{p}(\mu)-T\right| \leq 1}} \frac{d_{p}^{*}(\mu)}{\left|s-N+i r_{p}(\mu)\right|} \leq \sum_{\substack{\mu \in S_{p} \\
\left|r_{p}(\mu)-T\right| \leq 1}} \frac{d_{p}^{*}(\mu)}{r_{p}(\mu)+T}=O\left(T^{n-2}\right) \\
\sum_{\substack{\mu \in S_{p} \\
\left|r_{p}(\mu)-T\right| \leq 1}} \frac{d_{p}^{*}(\mu)}{\left(r_{p}(\mu)\right)^{2}+\alpha_{2}^{2}} \leq \sum_{\substack{\mu \in S_{p} \\
\left|r_{p}(\mu)-T\right| \leq 1}} \frac{d_{p}^{*}(\mu)}{(T-1)^{2}}=O\left(T^{n-3}\right) .
\end{aligned}
$$

This completes the proof

To compare the analogous considerations for the Riemann zeta function, we refer to Landau [19] and Titchmarsh [26], for the case $n=2, p=0$ cf. Hejhal [14]. We easily get the following conclusion.

Proposition 41: For $0<\epsilon<1, s=N+\sigma+i T, \sigma \geq \epsilon$ and $T \geq 100$, we have $\Psi_{p}(s)=O\left(\frac{T^{n-1}}{e}\right)$.

Proof: In the case $\sigma \leq N+1$ the assertion is a consequence of $\frac{1}{\left|\rho-N+i_{p}(\mu)\right|} \leq \frac{1}{e}$, the estimation $\mathcal{N}_{p}(T+1)-\mathcal{N}_{p}(T-1)=O\left(T^{n-1}\right)$ (which follows from Proposition 34) and Proposition 40 . In the case $\sigma \geq N+1$ we have $\Psi_{p}(s)=O(1)$ by Lemma 15 व

We need the following Phragmén-Lindelöf principle, stated in Landau [19, Satz 405].

Proposition 42: Suppose $\beta>\alpha, T_{0}>0$ and let the function $f(s)$ be holomorphic in the half strip $\alpha \leq \sigma^{*} \leq \beta, T \geq T_{0}$ with $s=\sigma^{*}+i T$. Further on, we suppose $f(s)=O\left(T^{\alpha}\right)$ for $\sigma^{*}=\alpha$ and $f(s)=O\left(T^{\beta}\right)$ for $\sigma^{*}=\beta$ and $|f(s)|=O\left(T^{c_{2}}\right)$ in the used half strip. Then it follows

in the introduced half strip.

$$
|f(s)|=O\left(T^{a \frac{\beta-\sigma^{\bullet}}{\beta-\alpha}+b \frac{\sigma^{\bullet}-a}{\beta-\alpha}}\right)
$$

Proposition 43: Suppose $0<\epsilon<1, s=N+\sigma+i T, \sigma \geq \epsilon$ and $T \geq 100$. Then we can state $\Psi_{p}(s)=O\left(\frac{1}{e} T^{2 \max [0,-\sigma+N+c]}\right)$.

Proof: We use Proposition 42 with $f(s)=\epsilon \Psi_{p}(s+\epsilon)$ and the half strip $N \leq R e s \leq$ $2 N, I m s \geq 100$. The function $f(s)$ is holomorphic in this half strip by Proposition 23 . As a consequense of Proposition 41 we have

$$
f(s)=O\left(\frac{1}{\epsilon}|\operatorname{Ims}|^{n-1}\right) \quad \text { and } \quad f(N+i T)=O\left(T^{n-1}\right) .
$$

Lemma 15 implies $f(2 N+i T)=O(1)$. We get the assertion for $R e s \leq 2 N$. In the case $\operatorname{Re} s \geq 2 N$ we only have to apply Lemma 15 again 


\section{Proof of Theorem B}

We are generalizing the proof for $n=2, p=0$ given by Hejhal [14], but we have much more technical problems. Using the length spectrum of the considered compact space form $V$ we introduce

$$
\Lambda_{p}(\omega)=l(\omega) \sigma(\omega) e_{p}(\omega) e^{l(\omega) N} .
$$

Then we can write (62) (valid for $R e s>2 N$ ) in the form

$$
\Psi_{p}(s)=\kappa_{p} \sum_{\omega \in \Omega} \Lambda_{p}(\omega) e^{-l(\omega) s} .
$$

Using a cut-off number $t$ with

$$
t \geq 20
$$

we define

$$
\Psi_{p}^{[t]}(s)=\frac{1}{2 \pi i} t^{1-n} \int_{R e \xi=\alpha} X(\xi, s, t) \Psi_{p}(\xi) d \xi
$$

with

$$
X(\xi, s, t)=\frac{\left(e^{(\xi-s) t}-e^{2(\xi-s) t}\right)^{n-1}}{(\xi-s)^{n}}
$$

and

$$
\alpha=\max (4 N, 2 N+\operatorname{Res}) .
$$

For $\operatorname{Re} \xi=\alpha$ we have $\Psi_{p}(\xi)=O(1)$ by Lemma 15 and thereby the integral in (109) exists. For $s=\sigma^{*}+i T$ (as above) we suppose

$$
\begin{gathered}
0 \leq \sigma^{*} \leq 4 N \\
T \geq 50, \quad T+k \notin\left\{r_{p}(\mu): \mu \in S_{p}\right\} \quad \text { for } k \in \mathrm{Z},|k| \leq 15 .
\end{gathered}
$$

For further considerations we use numbers

$$
\begin{aligned}
\sigma_{1} & \in \mathbf{R} \text { with } N<\sigma_{1}<4 N / 3 \\
F & =T+10 \\
G & =T-10 \\
A & \in\{k+1 / 2: k \in \mathbf{Z}, k \geq N\}
\end{aligned}
$$

We denote by $R(A, s)$ the rectangle defined by the points $-A-i F, \alpha-i F, \alpha+i F$ and $-A+i F$. We remark that $F, G$ and $\alpha$ are depending on $s$. An interesting background of the definition of $\Psi_{p}^{(t)}(\omega)$ is the fact that it will turn out to be a sum which is similar to (107), but finite. More precisely, we get

Proposition 44: For $R e s>2 N$ we obtain

$$
\Psi_{p}^{[t]}(s)=\kappa_{p} \sum_{\omega \in \Omega} \Lambda_{p}^{t}(\omega) e^{-l(\omega) s}
$$

with

$$
\Lambda_{p}^{t}(\omega)=\sum_{k=0}^{n-1} \frac{(-1)^{k}}{k !(n-1-k) !}\left\{(n-1+k)-\frac{l(\omega)}{t}\right\}_{+}^{n-1} \Lambda_{p}(\omega)
$$


Thereby we use $\nu_{+}^{n-1}= \begin{cases}\nu^{n-1} & \text { for } \nu>0 \\ 0 & \text { for } \nu \leq 0\end{cases}$

Proof: The definitions imply

$$
\begin{aligned}
\Psi_{p}^{[t]}(s) & =\frac{\kappa_{p}}{2 \pi i} t^{1-n} \sum_{\omega \in \Omega} \int_{R e \xi=\alpha} X(\xi, s, t) e^{-l(\omega) \xi} d \xi \Lambda_{p}(\omega) \\
& =\frac{\kappa_{p}}{2 \pi i} t^{1-n} \sum_{\omega \in \Omega} \int_{R e u=\alpha_{-\sigma^{\circ}}}\left(e^{u t}-e^{2 u t}\right)^{n-1} u^{-n} e^{-l(\omega) u} d u \Lambda_{p}(\omega) e^{-l(\omega) .} .
\end{aligned}
$$

In order to calculate the integral we use the Cauchy residue theorem. Let $K_{\rho}^{0}$ denote the straight line from $\alpha-\sigma^{*}-i \rho$ to $\alpha-\sigma^{*}+i \rho$. We denote the right curcular arc of the circle with center 0 , radius $\rho$ given by the chord $K_{\rho}^{0}$ by $K_{\rho}^{R}$. Analogously we define the left circular arc $K_{\rho}^{L}$. We can state

$$
\left(e^{u t}-e^{2 u t}\right)^{n-1} u^{-n}=\sum_{k=0}^{n-1}(-1)^{k}\left(\begin{array}{c}
n-1 \\
k
\end{array}\right) e^{(n-1+k) u t} u^{-n} .
$$

We consider the integrals

$$
I_{k, l(\omega)}^{*}=\int_{R e u=\alpha-\sigma^{*}} e^{(n-1+k) u t} u^{-n} e^{-l(\omega) u} d u .
$$

For $0<(n-1+k) t<l(\omega)$ the integrand $e^{(n-1+k) u t} u^{-n} e^{-l(\omega) u}$ is holomorphic with respect to $n$ in the domain bounded by $K_{\rho}^{0}$ and $K_{\rho}^{R}$. For $u \in K_{\rho}^{R}$ we obtain

$$
\left|e^{(n-1+k) u t} u^{-n} e^{-l(\omega) u}\right| \leq \rho^{-n}
$$

and the arc length of $K_{p}^{R}$ is smaller then $\pi \rho$. It follows

$$
\lim _{\rho \rightarrow \infty} \int_{K_{\rho}^{R}} e^{(n-1+k) u t} u^{-n} e^{-l(\omega) u} d u=0 .
$$

Now the residue theorem implies

$$
\int_{R e u=\alpha-\sigma^{*}} e^{(n-1+k) u t} u^{-n} e^{-l(\omega) u} d u=0 .
$$

For $l(\omega) \leq(n-1+k) t$ the integrand $e^{(n-1+k) u t} u^{-n} e^{-l(\omega) u}$ is holomorphic in the domain bounded by $K_{p}^{0}$ and $K_{\rho}^{L}$ with the exception of the pole in $u=0$. For $u \in K_{\rho}^{L}$ we can estimate

$$
\left|e^{(n-1+k) u t} u^{-n} e^{-l(\omega) u}\right| \leq e^{[(n-1+k) t-l(\omega)]\left(\alpha-\sigma^{*}\right)} \rho^{-n} .
$$

The arc length of $K_{\rho}^{L}$ is smaller then $2 \pi \rho$ and thereby we get

$$
\lim _{\rho \rightarrow \infty} \int_{K_{\rho}^{L}} e^{(n-1+k) u t} u^{-n} e^{-l(\omega) u} d u=0 .
$$

The residue of the integrand in $u=0$ follows from the equation

$$
e^{((n-1+k) t-l(\omega)] u} u^{-n}=u^{-n}+\ldots+\frac{1}{(n-1) !}[(n-1+k) t-l(\omega)]^{n-1} u^{-1}+\ldots
$$


For $l(\omega) \leq(n-1+k) t$ the application of the Cauchy residue theorem gives

$$
\int_{R e u=\alpha-\alpha^{\circ}} e^{(n-1+k) u t} u^{-n} e^{-l(\omega) u} d u=\frac{2 \pi i}{(n-1) !}((n-1+k) t-l(\omega))^{n-1} .
$$

Summarizing the results gives

$$
\begin{aligned}
\int_{R e u=\alpha-\sigma^{-}} e^{(n-1+k) u t} u^{-n} e^{-l(\omega) u} d u \\
=\sum_{k=0}^{n-1}(-1)^{k}\left(\begin{array}{c}
n-1 \\
k
\end{array}\right) \frac{2 \pi i}{(n-1) !}\{(n-1+k) t-l(\omega)\}_{+}^{n-1} .
\end{aligned}
$$

Now the assertion follows from equation (120)

We have

$$
\Lambda_{p}^{t}(\omega)=0 \text { for } l(\omega) \geq 2(n-1) t .
$$

It follows that the sum in (118) is finite in contrast to the sum in (107). We use the fact that we have found an analytic continuation of $\Psi_{p}(\xi)$ (cf. Proposition 23) and apply the Cauchy integral theorem to the function $X(\xi, s, t) \Psi_{p}(\xi)$ and the domain $R(A, s)$ :

$$
\begin{aligned}
\frac{1}{2 \pi i} \int_{\alpha-i F}^{\alpha+i F} X(\xi, s, t) \Psi_{p}(\xi) d \xi \\
\quad=\frac{1}{2 \pi i} \int_{-A-i F}^{-A+i F} X(\xi, s, t) \Psi_{p}(\xi) d \xi+\frac{1}{2 \pi i} \int_{-A+i F}^{\alpha+i F} X(\xi, s, t) \Psi_{p}(\xi) d \xi \\
\quad-\frac{1}{2 \pi i} \int_{-A-i F}^{\alpha-i F} X(\xi, s, t) \Psi_{p}(\xi) d \xi+\sum_{\xi \in \operatorname{Pl}_{A, 0}} \operatorname{Res}\left(X(\xi, s, t) \Psi_{p}(\xi)\right) .
\end{aligned}
$$

Thereby $P_{0 l_{A},}$ denotes the set of simple poles of the function $X(\xi, s, t) \Psi_{p}(\xi)$ with respect to the variable $\xi$ within the rectangle $R(A, s)$. Because of (113), (117) and Proposition 23 there are no poles situated on the boundary of $R(A, s)$. Our next task will be the estimation of the terms on the right-hand side of (122).

Proposition 45: If we suppose (108) - (117) we get

$$
\frac{1}{2 \pi i} \int_{-A-i F}^{-A+i F} X(\xi, s, t) \Psi_{p}(\xi) d \xi=O\left(\left[1+(F / A)^{n-1}\right] e^{-(n-1)\left(A+\sigma^{*}\right) t}\right) .
$$

Proof: We consider the integrals

$$
\begin{aligned}
& L_{1}=\int_{-A-i F}^{-A-i} Y(\xi, s, t) \Psi_{p}(\xi) d \xi \\
& L_{2}=\int_{-A-i}^{-A+i} Y(\xi, s, t) \Psi_{p}(\xi) d \xi \\
& L_{3}=\int_{-A+i}^{-A+i F} Y(\xi, s, t) \Psi_{p}(\xi) d \xi
\end{aligned}
$$


with

$$
Y(\xi, s, t)=\frac{e^{\xi-s) t}}{(\xi-s)^{n}} .
$$

Using the functional equation of Proposition 25 we obtain

$$
L_{1}=\int_{-A-i F}^{-A-i} Y(\xi, s, t)\left[-\Psi_{p}(2 N-\xi)+\Phi_{p}(\xi-N)\right] d \xi
$$

If we suppose (117) and apply Lemma 15 we get the estimation $\left|\Psi_{p}(2 N-s)\right|=O(1)$ for Re $s=-A, A \geq \frac{3}{2}$. Applying (87) we obtain

$$
\left|-\Psi_{p}(2 N-s)+\Phi_{p}(s-N)\right|= \begin{cases}O\left(s^{n-1} \tan (\pi s)\right) & \text { for } n \text { even } \\ O\left(s^{n-1}\right) & \text { for } n \text { odd }\end{cases}
$$

For $s=k+\frac{1}{2}+i T, k \in \mathbf{Z}, T \geq 0$ we have

$$
\tan (\pi s)=\tan \left(\pi\left(k+\frac{1}{2}+i T\right)\right)=-\cot (\pi i T)=i \cot (\pi T)=i \frac{1+e^{-2 \pi T}}{1-e^{-2 \pi T}}=O(1)
$$

and thereby

$$
\left|-\Psi_{p}(2 N-\xi)+\Phi_{p}(\xi-N)\right|=O\left(\xi^{n-1}\right)
$$

for $\xi \in[-A-i F,-A-i]$. It follows $L_{1}=O\left(L_{1}^{\prime}\right)$ with

$$
L_{1}^{\prime}=\int_{-A-i F}^{-A-i}|Y(\xi, s, t)||\xi|^{n-1} d \xi=\int_{-F}^{-1} \frac{e^{-\left(A+\sigma^{*}\right) t}}{\left(\left(A+\sigma^{*}\right)^{2}+(\eta+T)^{2}\right)^{n / 2}}\left(A^{2}+\eta^{2}\right)^{\frac{n-1}{2}} d \eta
$$

Since $f(r)=r^{(n-1) / 2}$ is a convex function for $n \geq 3$, we get $\left(A^{2}+\eta^{2}\right)^{(n-1) / 2} \leq$ $2^{(n-3) / 2}\left(A^{n-1}+\eta^{n-1}\right)$. For $n=2$ we use $\left(A^{2}+\eta^{2}\right)^{1 / 2} \leq A+\eta$. We conclude $L_{1}^{\prime}=O\left(L_{1}^{\prime \prime}\right)$ with

$$
\begin{aligned}
L_{1}^{\prime \prime} & =\int_{1}^{F} \frac{e^{-\left(A+\sigma^{*}\right) t}}{\left[\left(A+\sigma^{*}\right)^{2}+(\eta+T)^{2}\right]^{n / 2}}\left(A^{n-1}+\eta^{n-1}\right) d \eta \\
& \leq\left(A^{n-1}+F^{n-1}\right) e^{-\left(A+\sigma^{*}\right) t} \int_{1}^{F} \frac{1}{\left[\left(A+\sigma^{*}\right)^{2}+(\eta+T)^{2}\right]^{n / 2}} d \eta \\
& \leq \frac{A^{n-1}+F^{n-1}}{A^{n-2}} e^{-\left(A+\sigma^{*}\right) t} \int_{1}^{F} \frac{1}{\left(A+\sigma^{*}\right)^{2}+(\eta+T)^{2}} d \eta, \\
L_{1}^{\prime \prime} & =O\left(\left[1+\left(\frac{F}{A}\right)^{n-1}\right] e^{-\left(A+\sigma^{*}\right) t}\right) .
\end{aligned}
$$

In order to get the last equation, we have used $\int_{1}^{F} \frac{1}{\left(A+\sigma^{\circ}\right)^{2}+(\eta+T)^{2}} d \eta=O\left(\frac{1}{A}\right)$. It follows that

$$
L_{1}=O\left(\left[1+\left(\frac{F}{A}\right)^{n-1}\right] e^{-\left(A+\sigma^{*}\right) t}\right)
$$


We get the same estimation for $L_{3}$.

It remains to estimate $L_{2}$. For that purpose we apply again the functional equation of Proposition 25 and the estimation (126):

$$
\begin{aligned}
L_{2} & =O\left(\int_{-A-i}^{-A+i}|Y(\xi, s, t)| \xi^{n-1} d \xi\right) \\
& =e^{-\left(A+\sigma^{*}\right) t} O\left(\int_{-1}^{1} \frac{\left(A^{2}+\eta^{2}\right)^{\frac{n-1}{2}}}{\left[\left(A+\sigma^{*}\right)^{2}+(\eta+T)^{2}\right]^{n / 2}} d \eta\right) \\
& =e^{-\left(A+\sigma^{*}\right) t} O\left(\frac{A^{n-1}}{A^{n}}\right) .
\end{aligned}
$$

So we have proved

$$
\int_{-A-i F}^{-A+i F} Y(\xi, s, t) \Psi_{p}(\xi) d \xi=O\left(\left[1+\left(\frac{F}{A}\right)^{n-1}\right] e^{-\left(A+\sigma^{*}\right) t}\right) .
$$

We use this estimation for every term of the binomial expansion of $\left(e^{(\varepsilon-s) t}-e^{2(\xi-s) t}\right)^{n-1}$ to complete the proof

Proposition 46: If we suppose (108) - (117), we get

(i) $\int_{-A+i F}^{-2 N+i F}\left|X(\xi, s, t) \Psi_{p}(\xi)\right||d \xi|=O\left(\frac{F^{n-1}}{t} e^{-\left(2 N+\sigma^{*}\right) t}\right)$

(ii) $\int_{4 N+i F}^{\alpha+i F}\left|X(\xi, s, t) \Psi_{p}(\xi)\right||d \xi|=O\left(e^{2(n-1)\left(a-\sigma^{\bullet}\right) z}\right)$.

Proof: We consider

$$
L_{4}=\int_{-A+i F}^{-2 N+i F}\left|Y(\xi, s, t) \Psi_{p}(\xi)\right||d \xi| .
$$

The functional equation of Proposition 25 implies

$$
L_{4}=\int_{-A+i F}^{-2 N+i F}\left|Y(\xi, s, t)\left[\Psi_{p}(2 N-\xi)+\Phi_{p}(\xi-N)\right]\right||d \xi| .
$$

We will apply (125) and suppose $-A-N \leq r \leq-3 N$ for $s=r+i F$. Then we get

$$
\tan (\pi s)=\tan (\pi(r+i F))=-i \tanh (\pi(r i-F))=-i \frac{e^{2 \pi(r i-F)}-1}{e^{2 \pi(r i-F)}+1}
$$

Using (113) and (115) we obtain $|\tan (\pi s)|<2$ and thereby $\left|\Phi_{p}(\xi-N)\right|=O\left(\xi^{n-1}\right)$. Because of

$$
\operatorname{Re}(2 N-\xi)>2 N+\frac{1}{2} \text { for } \xi \in[-A+i F,-2 N+i F]
$$


we get $\Psi_{p}(2 N-\xi)=O(1)$. It follows

$$
L_{4}=O\left(\int_{-A+i F}^{-2 N+i F}|Y(\xi, s, t)||\xi|^{n-1}|d \xi|\right) .
$$

By (108) and (115) we get

$$
L_{4}=O\left(F^{n-1} \int_{-A}^{-2 N} e^{\left(\eta-0^{*}\right) t}|d \eta|\right) \text { and } L_{4}=O\left(\frac{F^{n-1}}{t} e^{\left(-2 N-\sigma^{*}\right) t}\right) .
$$

If we use the binomial expansion of $\left(e^{(t-a) t}-e^{2(t-o) t}\right)^{n-1}$, we get the assertion (i). For $0 \leq \sigma^{*} \leq 2 N$ we have $\alpha=4 N$ and consequently the upper and lower bounds of the integral in (ii) are the same. So let us suppose $2 N \leq \sigma^{*} \leq 4 N$. Then we get

$$
L_{5}=\int_{1 N+i F}^{a+i F}\left|X(\xi, s, t) \Psi_{p}(\xi)\right||d \xi| \leq \int_{\mathcal{L}}^{\alpha} \frac{e^{2(n-1)\left(\eta-\sigma^{*}\right) t}}{10^{n}}|O(1)| d \eta=O\left(e^{2(n-1)\left(\alpha-\sigma^{*}\right) t}\right) .
$$

Thereby we have used Lemma 15

Assumption 47: We suppose that the cutting-off parameter $t$ and the imaginary part $T=I m s$ of the considered complex parameter $s$ satisfy the inequality $e^{t} \leq T^{2}$.

Proposition 48: We suppose $\sigma_{1}=N+\epsilon, 0<\epsilon<1 / 10,(108)-(117), \sigma_{1} \leq \sigma^{*}$ and Assumption 47. Then it holds

(i) $\int_{N+c+i F}^{4 N+i F}\left|Y(\xi, s, t) \Psi_{p}(\xi)\right||d \xi|=O\left(\frac{F^{2 N}}{e} e^{\left(\sigma_{1}-\sigma^{\bullet}\right) t}\right)+O\left(\frac{1}{c} e^{\left(4 N-\sigma^{\bullet}\right) t}\right)$

(ii) $\int_{-2 N+i F}^{N-\varepsilon+i F}\left|Y(\xi, s, t) \Psi_{p}(\xi)\right||d \xi|=O\left(\frac{F^{2 N}}{e} e^{\left(N-\varepsilon-\sigma^{*}\right) t}\right)$.

Proof: For $\xi=\eta+i F, s=\sigma^{*}+i T$ we define

$$
L_{6}=\int_{N+c+i F}^{4 N+i F}\left|Y(\xi, s, t) \Psi_{p}(\xi)\right||d \xi|
$$

and get

$$
L_{6} \leq 10^{-n} \int_{N+e}^{4 N} e^{\left(\eta-\sigma^{*}\right) t}\left|\Psi_{p}(\eta+i F)\right| d \eta
$$

By using Proposition 43, it follows

$$
\left|\Psi_{p}(\eta+i F)\right|=O\left(\frac{1}{\epsilon} F^{2 \max (0,-\eta+2 N+c)}\right) \text { for } \eta \in[N+\epsilon, 4 N] .
$$

We easily deduce that

$$
L_{6}=O\left(\frac{1}{\epsilon} \int_{N+e}^{4 N} e^{\left(\eta-\sigma^{*}\right) t} F^{2 \max (0,-\eta+2 N+\varepsilon)} d \eta\right)
$$


Spectral Estimates for Compact Hyperbolic Space Forms

283

$$
\begin{aligned}
& =O\left(\frac{1}{\epsilon} \int_{N+\varepsilon}^{2 N+\epsilon} e^{\left(\eta-\sigma^{*}\right) t} F^{2(-\eta+2 N+c)} d \eta\right)+O\left(\frac{1}{\epsilon} \int_{2 N+\varepsilon}^{4 N} e^{\left(\eta-\sigma^{*}\right) t} d \eta\right) \\
& =O\left(\frac{1}{\epsilon} e^{-\sigma^{*} t} F^{2(2 N+c)} \int_{N+c}^{2 N+c}\left(e^{t} / F^{2}\right)^{\eta} d \eta\right)+O\left(\frac{1}{\epsilon} e^{\left(4 N-\sigma^{*}\right) t}\right) .
\end{aligned}
$$

Using (115) and Assumption 47, we obtain $e^{i} \leq T^{2} \leq F^{2}$. Accordingly we get

$$
\begin{aligned}
L_{6} & =O\left(\frac{1}{\epsilon} e^{-\sigma^{*} t} F^{2(2 N+c)}\left(\frac{e^{t}}{F^{2}}\right)^{N+\epsilon}\right)+O\left(\frac{1}{\epsilon} e^{\left(4 N-\sigma^{*}\right) t}\right) \\
& =O\left(\frac{1}{\epsilon} F^{2 N} e^{\left(\sigma_{1}-\sigma^{*}\right) t}\right)+O\left(\frac{1}{\epsilon} e^{\left(4 N-\sigma^{*}\right) t}\right) .
\end{aligned}
$$

This proves (i).

In order to study

$$
L_{7}=\int_{-2 N+i F}^{N-\imath+i F}\left|Y(\xi, s, t) \Psi_{p}(\xi)\right||d \xi|
$$

we use Proposition 25 and get

$$
L_{7}=O\left(\int_{-2 N+i F}^{N-c+i F} e^{\left(\eta-\sigma^{*}\right) t}\left|-\Psi_{p}(2 N-\xi)+\Phi_{p}(\xi-N)\right||d \xi|\right) .
$$

By Proposition 41 we obtain

$$
\left|\Psi_{p}(2 N-\xi)\right|=O\left(\frac{1}{\epsilon} F^{n-1}\right) \quad \text { for } \xi \in[-2 N+i F, N-\epsilon+i F] .
$$

Using $\left|\Phi_{p}(\xi-N)\right|=O\left(F^{n-1}\right)$, it follows

$$
L_{7}=O\left(\left(\frac{1}{\epsilon} F^{n-1}+F^{n-1}\right) \int_{-2 N}^{N-e} e^{\left(\eta-\sigma^{*}\right) t} d \eta\right)=O\left(\frac{F^{2 N}}{\epsilon} e^{\left(N-\epsilon-\sigma^{\bullet}\right) t}\right) .
$$

Thus also statement (ii) is proved

Using Proposition 48 a quick calculation shows

Proposition 49: We suppose $\sigma_{1}=N+\epsilon, 0<\epsilon<1 / 10, \sigma_{1} \leq \sigma^{*},(108)-(117)$ and Assumption 47. Then it holds

(i) $\int_{N+\varepsilon+i F}^{4 N+i F}\left|X(\xi, s, t) \Psi_{p}(\xi)\right||d \xi|=O\left(\frac{F^{2 N}}{e} e^{\left(\sigma_{1}-\sigma^{*}\right) t}\right)+O\left(\frac{1}{e} e^{\left(4 N-\sigma^{*}\right) t}\right)$

(ii) $\int_{-2 N+i F}^{N-e+i F}\left|X(\xi, s, t) \Psi_{p}(\xi)\right||d \xi|=O\left(\frac{F^{2 N}}{e} e^{\left(N-\iota-\sigma^{*}\right) t}\right)$.

Proposition 50: We suppose $\sigma_{1}=N+\epsilon, 0<\epsilon<1 / 10, \sigma_{1} \leq \sigma^{*},(108)-(117)$ and Assumption 47. Then it holds

$$
\int_{N-i+i F}^{N+e+i F} Y(\xi, s, t) \Psi_{p}(\xi) d \xi=O\left(F^{2 N} e^{\left(\sigma_{1}-\sigma^{\bullet}\right) t}\right)
$$


Proof: Proposition 40 with $\xi=\eta+i F$ implies

$$
\Psi_{p}(\xi)=O\left(F^{n-1}\right)+\kappa_{p} \sum_{\substack{\mu \in S_{p} \\\left|r_{p}(\mu)-F\right| \leq 1}} d_{p}^{*}(\mu) \frac{1}{\xi-N-i r_{p}(\mu)}
$$

for $\xi \in[N-\epsilon+i F, N+\epsilon+i F]$. We get

$$
\begin{aligned}
& L_{8}=\int_{N-\epsilon+i F}^{N+c+i F} Y(\xi, s, t) \Psi_{p}(\xi) d \xi \\
& =\int_{N-e+i F}^{N+c+i F} Y(\xi, s, t) O\left(F^{n-1}\right) d \xi \\
& +\kappa_{p} \sum_{\substack{\mu \in S_{p} \\
\left|r_{p}(\mu)-F\right| \leq 1}} d_{p}^{=}(\mu) \int_{N-c+i F}^{N+c+i F} Y(\xi, s, t) \frac{1}{\xi-N-i r_{p}(\mu)} d \xi .
\end{aligned}
$$

It holds $|\xi-s| \geq 10$ and thereby we get

$$
\int_{N-\epsilon+i F}^{N+e+i F} Y(\xi, s, t) O\left(F^{n-1}\right) d \xi=O\left(\epsilon e^{\left(\sigma_{1}-\sigma^{*}\right) t} F^{n-1}\right)
$$

In order to analyze

$$
L_{8, \mu}=\int_{N-c+i F}^{N+e+i F} Y(\xi, s, t) \frac{1}{\xi-N-i r_{p}(\mu)} d \xi
$$

we use the Cauchy integral theorem transforming the path of integration into a semicircular. We denote the upper semi-circular above $[N-\epsilon+i F, N+\epsilon+i F]$ by $H^{+}$and the corresponding lower semi-circular by $H^{-}$. Since (113) we have $r_{p}(\mu) \neq F$. Then the function $\frac{Y(\xi, s, t)}{\xi-N-i r_{p}(\mu)}$ is holomorphic with respect to $\xi$ in the domain bounded by $H^{+}$and $H^{-}$because of $\xi \neq s$. For $r_{p}(\mu)>F$ and $r_{p}(\mu)<F$ we use $H^{-}$and $H^{+}$, respectively as the new path of integration. Using $\epsilon<1 / 10$ we get $|\xi-s|>9$ and $\left|\xi-N-i r_{p}(\mu)\right|>\epsilon$. It follows

$$
L_{8, \mu}=O\left(e^{\left(\sigma_{1}-\sigma^{*}\right) t}\right) .
$$

Thereby the $O$-term is independent of $\mu$ and $T$. From (127) - (129) we get

$$
L_{8}=O\left(e^{\left(\sigma_{1}-\sigma^{*}\right) t} F^{n-1} \epsilon\right)+\sum_{\left|r_{p}(\mu)-F\right| \leq 1} O\left(d_{p}^{*}(\mu) e^{\left(\sigma_{1}-\sigma^{*}\right) t}\right) .
$$

Proposition 34 implies $\sum_{\left|r_{p}(\mu)-F\right| \leq 1} d_{p}^{*}(\mu)=O\left(F^{n-1}\right)$ and we easily obtain the assertion

Of course, we again get the corresponding result using $X(\xi, s, t)$ instead of $Y(\xi, s, t)$. 
Proposition 51: We suppose $\sigma_{1}=N+\epsilon, 0<\epsilon<1 / 10, \sigma_{1} \leq \sigma^{*},(108)-(117)$ and Assumption 4\%. Then it holds

$$
\int_{N-\epsilon+i F}^{N+\imath+i F} X(\xi, s, t) \Psi_{p}(\xi) d \xi=O\left(F^{2 N} e^{\left(\sigma_{1}-\sigma^{*}\right) t}\right)
$$

We have described the integrals on the right-hand side of (122) and get information about the term on the left-hand side of (122).

Proposition 52: We suppose $\sigma_{1}=N+\epsilon, \quad 0<\epsilon<1 / 10, \sigma_{1} \leq \sigma^{\circ}$, (108) - (117) and Assumption \&7. Then it holds

$$
\begin{aligned}
& \frac{1}{2 \pi i} \int_{\alpha-i F}^{\alpha+i F} X(\xi, s, t) \Psi_{p}(\xi) d \xi \\
& \quad=O\left(\left[1+\left(\frac{F}{A}\right)^{2 N}\right] e^{-2 N\left(A+\sigma^{*}\right) t}\right)+O\left(\frac{F^{2 N}}{t} e^{-\left(2 N+\sigma^{*}\right) t}\right) \\
& \quad+O\left(e^{4 N\left(\alpha-\sigma^{*}\right) t}\right)+O\left(\frac{1}{\sigma_{1}^{-}-N} e^{4 N\left(4 N-\sigma^{*}\right) t}\right) \\
& \quad+O\left(\frac{F^{2 N}}{\sigma_{1}-N} e^{\left(\sigma_{1}-\sigma^{\bullet}\right) t}\right)+\sum_{\bullet \in \operatorname{Pol}_{A, 0}} \operatorname{Res}\left(X(\xi, s, t) \Psi_{p}(\xi)\right)
\end{aligned}
$$

Proof: Proposition 45 states

$$
\frac{1}{2 \pi i} \int_{-\Lambda-i F}^{-A+i F} X(\xi, s, t) \Psi_{p}(\xi) d \xi=O\left(\left[1+\left(\frac{F}{A}\right)^{2 N}\right] e^{-2 N\left(A+\sigma^{-0}\right) \xi}\right) .
$$

Further on, we apply Propositions 46, 49 and 51:

$$
\begin{aligned}
& \frac{1}{2 \pi i} \int_{-\Lambda+i F}^{\alpha+i F} X(\xi, s, t) \Psi_{p}(\xi) d \xi \\
& \quad=O\left(\frac{F^{2 N}}{t} e^{-\left(2 N+\sigma^{*}\right) t}\right)+O\left(e^{4 N\left(\alpha-\sigma^{*}\right) t}\right) \\
& \quad+O\left(\frac{1}{\sigma_{1}-N} e^{4 N\left(4 N-\sigma^{*}\right) t}\right)+O\left(\frac{F^{2 N}}{\sigma_{1}-N} e^{\left(\sigma_{1}-\sigma^{*}\right) t}\right)
\end{aligned}
$$

There are similar calculations for $\int_{-A-i F}^{\alpha-i F}$ instead of $\int_{-A+i F}^{\alpha+i F}$. The proposition is completed by a simple application of the Cauchy integral theorem $\square$

Proposition 53: We suppose $\sigma_{1}=N+\epsilon, 0<\epsilon<1 / 10, \sigma_{1} \leq \sigma^{*},(108)-(117)$ and Assumption 47. Then it holds

$$
\frac{1}{2 \pi i} t^{1-n} \int_{a-i F}^{a+i F} X(\xi, s, t) \Psi_{p}(\xi) d \xi
$$




$$
\begin{aligned}
= & O\left(t^{1-n} e^{4 N\left(a-\sigma^{*}\right) t}\right)+O\left(\frac{t^{1-n}}{\sigma_{1}-N} e^{4 N\left(4 N-\sigma^{\bullet}\right) t}\right) \\
& +O\left(F^{2 N_{t^{-n}}} e^{-\left(2 N+\sigma^{*}\right) t}\right)+O\left(\frac{F^{2 N} t^{1-n}}{\sigma_{1}-N} e^{4 N\left(\sigma_{1}-\sigma^{-}\right) t}\right) \\
& +t^{1-n} \sum_{k=1}^{\infty} v_{k} X(-k, s, t)+t^{1-n} \sum_{k=1}^{M_{p}} r_{k} X\left(s_{k}, s, t\right) \\
& +t^{1-n} \sum_{\substack{\mu \in S_{p} \\
0<r_{p}(\mu) \leq F}} 2 \kappa_{p} d_{p}^{*}(\mu) X\left(s^{+}(\mu), s, t\right) \\
& +t^{1-n} \sum_{\substack{\mu \in S_{p} \\
0<r_{p}(\mu) \leq F}} 2 \kappa_{p} d_{p}^{*}(\mu) X\left(s^{-}(\mu), s, t\right)+(-1)^{n-1} \Psi_{p}(s) .
\end{aligned}
$$

Thereby we have used the residues of $\Psi_{p}(s)$ in the poles $\xi=-k$ given by $v_{k}=(-1)^{n / 2} 2(N+$ $k) \chi(V) P\left(-(N+k)^{2}\right)$ for $n$ even and $v_{k}=0$ for $n$ odd. The residues in the poles $\xi=$ $s_{1}, \ldots, s_{M_{p}}$ are denoted by $r_{k}\left(k=1, \ldots, M_{p}\right)$.

Proof: We use Proposition 52 and take the limit $A \rightarrow \infty$. We obtain

$$
\lim _{A \rightarrow \infty} O\left(\left[1+\left(\frac{F}{A}\right)^{2 N}\right] e^{-2 N\left(A+\sigma^{*}\right) t}\right)=0 .
$$

For the calculation of the residues we use Proposition 23. From the equation

$$
\begin{aligned}
{\left[e^{x t}-e^{2 x t}\right]^{n-1} x^{-n} } & =e^{(n-1) x t}\left[1-e^{x t}\right]^{n-1} x^{-n} \\
& =(1+\ldots)(-x t+\ldots)^{n-1} x^{-n} \\
& =(-t)^{n-1} x^{-1}+\ldots
\end{aligned}
$$

we conclude $\operatorname{Res}_{\xi=,} X(\xi, s, t)=(-t)^{n-1}$. We remark, that (112) and (113) ensure that $\xi=s$ is no pole of $\Psi_{p}(\xi)$

Proposition 54: We suppose (108) - (117) and Assumption 47. Then it holds

(i) $t^{1-n} \int_{a+i F}^{\alpha+i \infty}\left|X(\xi, s, t) \Psi_{p}(\xi)\right||d \xi|=O\left(t^{1-n} e^{4 N\left(\alpha-\sigma^{*}\right) t}\right)$

(ii) $t^{1-n} \int_{a-i \infty}^{\alpha-i F}\left|X(\xi, s, t) \Psi_{p}(\xi)\right||d \xi|=O\left(t^{1-n} e^{4 N\left(\alpha-\sigma^{*}\right) t}\right)$.

Proof: Since (111) and (112) we have $4 N \leq \alpha \leq 6 N$ and $\operatorname{Re}(\xi-s)=\alpha-\sigma^{*} \geq 2 N$ for $\xi \in[\alpha+i F, \alpha+i \infty)$. It follows

$$
\begin{aligned}
\int_{\alpha+i F}^{\alpha+i \infty}\left|X(\xi, s, t) \Psi_{p}(\xi)\right||d \xi| & \leq \int_{\alpha+i F}^{\alpha+i \infty} \frac{e^{4 N\left(\alpha-\sigma^{*}\right) t}}{|\xi-s|^{n}}|d \xi| O(1) \\
& \leq O\left(e^{4 N\left(\alpha-\sigma^{*}\right) t}\right) \int_{F}^{\infty} \frac{1}{\left[\left(\alpha-\sigma^{*}\right)^{2}+(\eta-T)^{2}\right]^{n / 2}} d \eta \\
& \leq O\left(e^{4 N\left(\alpha-\sigma^{*}\right) t}\right) .
\end{aligned}
$$

This proves (i), the proof of (ii) is similar 
Proposition 55: We suppose $\sigma_{1}=N+\epsilon, 0<\epsilon<1 / 10, \sigma_{1} \leq \sigma^{*},(108)-(117)$ and Assumption 47. Then it holds

$$
\begin{aligned}
\Psi_{p}^{[t]}(s)= & (-1)^{n-1} \Psi_{p}(s)+O\left(t^{1-n} e^{4 N\left(\alpha-\sigma^{*}\right) t}\right)+O\left(\frac{t^{1-n}}{\sigma_{1}-N} e^{4 N\left(4 N-\sigma^{*}\right) t}\right) \\
& +O\left(F^{2 N} t^{-n} e^{-\left(2 N+\sigma^{*}\right) t}\right)+O\left(\frac{F^{2 N} t^{1-n}}{\sigma_{1}-N} e^{\left(\sigma_{1}-\sigma^{*}\right) t}\right) \\
& +t^{1-n} \sum_{k=1}^{\infty} v_{k} X(-k, s, t)+t^{1-n} \sum_{k=1}^{M_{p}} r_{k} X\left(s_{k}, s, t\right) \\
& +t^{1-n} \sum_{\substack{\mu \in S_{p} \\
0<r p(\mu) \leq F}} 2 \kappa_{p} d_{p}^{*}(\mu) X\left(s^{+}(\mu), s, t\right) \\
& +t^{1-n} \sum_{\substack{\mu \in S_{p} \\
0<r p(\mu) \leq F}} 2 \kappa_{p} d_{p}^{*}(\mu) X\left(s^{-}(\mu), s, t\right) .
\end{aligned}
$$

Proof: The assertion is an immediate consequence of definition (109), Proposition 53 and Proposition 54

Our next aim is a simplification of the estimation of Proposition 55. It will turn out to be useful to suppose the following

Assumption 56: From now on we suppose

(i) $e^{t} \leq T^{\frac{1}{T N}}$

(ii) $\sigma_{1}=N+\frac{1}{t}$

(iii) $\sigma_{1} \leq \sigma^{*} \leq 4 N$.

We get part (ii) of Assumption 56 if we minimize $\frac{1}{\sigma_{1}-N} e^{\left(\sigma_{1}-\sigma^{\bullet}\right) t}$ with respect to $\sigma_{1}$.

Proposition 57: We suppose (108) - (117), Assumption 47 and 56. Then it holds

$$
(-1)^{n-1} \Psi_{p}(s)=\Psi_{p}^{[t]}(s)+S^{\#}+O\left(F^{2 N} e^{\left(N-\sigma^{\bullet}\right) t}\right)
$$

with

$$
S^{\#}=t^{1-n} \sum_{\substack{\mu \in S_{p} \\\left|r_{p}(\mu)-\tau\right| \leq 10}} 2 \kappa_{p} d_{p}^{*}(\mu) X\left(s^{+}(\mu), s, t\right)
$$

Proof: We first want to prove the assertion of Proposition 57 with

$$
\tilde{S}^{\#}=t^{1-n} \sum_{\substack{\mu \in S_{p} \\ 0 \leq p p(\mu) \leq F}} 2 \kappa_{p} d_{p}^{*}(\mu) X\left(s^{+}(\mu), s, t\right)
$$

instead of $S^{\#}$. In order to reach this goal we want to show that the O-estimates of Proposition 55 are included in the $O$-estimate of Proposition 57.

1. We consider $t^{1-n} e^{4 N\left(\alpha-\sigma^{*}\right) t}$. If we have $0 \leq \sigma^{*} \leq 2 N$, then we get $\alpha=4 N$ by (111) and therefore Assumption 56/(i) gives

$$
e^{\left(2 \alpha-\sigma^{*}-N\right) 2 N t} \leq T^{2 N} \text { and } e^{4 N\left(\alpha-\sigma^{*}\right) t} \leq e^{2\left(N-\sigma^{*}\right) N t} T^{2 N}
$$


and consequently

$$
t^{-2 N} e^{4 N\left(\alpha-\sigma^{*}\right) t} \leq T^{2 N} e^{2 N\left(N-\sigma^{*}\right) t} .
$$

For $2 N \leq \sigma^{*} \leq 4 N$ it holds $\alpha=2 N+\sigma^{*}$ and thereby

$$
e^{\left(3 N+\sigma^{*}\right) t} \leq T, \quad e^{\left(2 a-2 \sigma^{*}+\sigma^{*}-N\right)} \leq T, \quad t^{-2 N} e^{4 N\left(a-\sigma^{*}\right) t} \leq T^{2 N} e^{2 N\left(N-\sigma^{*}\right) t} .
$$

It follows $t^{-2 N} e^{4 N\left(a-\sigma^{*}\right) t} \leq O\left(T^{2 N} e^{2 N\left(N-\sigma^{*}\right) t}\right)$ and thereby

$$
t^{-2 N} e^{4 N\left(\alpha-\sigma^{*}\right) t}=O\left(T^{2 N} e^{\left(N-\sigma^{*}\right) t}\right) .
$$

2. We consider $\frac{1}{\sigma_{1}-N} t^{-2 N} \cdot e^{4 N\left(4 N-\sigma^{\circ}\right) t}$. According to Assumption 56/(ii) we have $\left(\sigma_{1}-N\right) t=1$ and therefore $\frac{1}{\sigma_{1}-N} t^{1-n} \leq 1$. Furthermore Assumption $56 /(\mathrm{i})$ implies $e^{4 N\left(4 N-\sigma^{*}\right) t} \leq T^{2 N} e^{2 N\left(N-\sigma^{*}\right) t}$. Consequently we get

$$
\frac{1}{\sigma_{1}-N} t^{1-n} e^{4 N\left(4 N-\sigma^{*}\right) t}=O\left(T^{2 N} e^{2 N\left(N-\sigma^{*}\right) t}\right)
$$

and

$$
\frac{1}{\sigma_{1}-N} t^{1-n} e^{4 N\left(4 N-0^{*}\right) t}=O\left(T^{2 N} e^{\left(N-\sigma^{*}\right) t}\right)
$$

3. We consider $F^{2 N} t^{-n^{\prime}} e^{-\left(2 N+\sigma^{*}\right) t}$. We immediately obtain

$$
F^{2 N} t^{-n} e^{-\left(2 N+\sigma^{*}\right) t}=O\left(T^{2 N} e^{\left(N-\sigma^{*}\right) t}\right)
$$

4. Assumption $56 /(\mathrm{ii})$ implies $e^{\left(\sigma_{1}-\sigma^{*}\right) t}=e^{\left(N-\sigma^{*}\right) t} e$ and consequently we get

$$
\frac{1}{\sigma_{1}-N} t^{1-n} F^{2 N} e^{\left(\sigma_{1}-\sigma^{\bullet}\right) t}=O\left(T^{2 N} e^{\left(N-\sigma^{\bullet}\right) t}\right) .
$$

5. Since $v_{k}=O\left(k^{n-1}\right)$ by Proposition 23, we obtain

$$
\begin{aligned}
\left|t^{1-n} \sum_{k=1}^{\infty} v_{k} X(-\dot{k}, s, t)\right| & =O\left(t^{1-n} \sum_{k=1}^{\infty} \frac{e^{-(n-1)\left(k+\sigma^{*}\right) t}}{|k+s|^{n}} k^{n-1}\right) \\
& =O\left(t^{1-n} e^{-(n-1) 0^{*}} \sum_{k=1}^{\infty} T^{-n} \cdot e^{-(n-1) k t}\right) \\
& =O\left(t^{1-n} T^{-n} e^{-(n-1) \sigma^{*} t}\right) .
\end{aligned}
$$

We apply

$$
t^{1-n} T^{-n} e^{-(n-1) \sigma^{*} t}=O\left(T^{2 N} e^{2 N\left(N-\sigma^{*}\right) t}\right)
$$

and get

$$
t^{1-n} \sum_{k=1}^{\infty} v_{k} X(-k, s, t)=O\left(T^{2 N} e^{\left(N-\sigma^{*}\right) t}\right) .
$$

6. We consider the poles $s_{k}\left(k=1, \ldots, M_{p}\right)$ of $\Psi_{p}(s)$ which have the imaginary part 0 (cf. Proposition 23). Then it holds $0 \leq R e s_{k} \leq 2 N$ and we have $0 \leq \operatorname{Re} s^{+}(\mu) \leq 2 N$ and

$$
\left|X\left(s_{k}, s, t\right)\right| \leq 2^{n-1} T^{-n}\left[e^{\left(2 N-\sigma^{\bullet}\right) t}+e^{4 N\left(2 N-\sigma^{\bullet}\right) t}\right] .
$$


From Assumption 56/(i) we get

$$
T^{-n} e^{\left(2 N-\sigma^{\bullet}\right) t}=O\left(T^{2 N} e^{\left(N-\sigma^{\bullet}\right) t}\right) \text { and } T^{-n} e^{4 N\left(2 N-\sigma^{*}\right) t}=O\left(T^{2 N} e^{\left(N-\sigma^{\bullet}\right) t}\right) .
$$

Consequently we obtain

$$
\left|T^{1-n} \sum_{k=1}^{M_{p}} r_{k} \frac{\left[e^{-\left(s_{k}-s\right) t}-e^{-2\left(s_{k}-s\right) t}\right]^{n-1}}{\left(s_{k}-s\right)^{n}}\right|=O\left(T^{2 N} e^{2 N\left(N-0^{\circ}\right) t}\right) .
$$

7. Further on we get

$$
\begin{aligned}
L_{9} & =\left|t^{1-n} \sum_{0 \leq r_{p}(\mu) \leq F} 2 \kappa_{p} d_{p}^{*}(\mu) X\left(s^{-}(\mu), s, t\right)\right| \\
& =O\left(\sum_{0 \leq r_{p}(\mu) \leq F} d_{p}^{*}(\mu) \frac{O\left(e^{\left(N-\sigma^{*}\right) t}\right)}{\left|s^{-}(\mu)-s^{n}\right|^{n-1}}\right) \\
& =\left(\sum_{0 \leq r_{p}(\mu) \leq F} d_{p}^{*}(\mu) t^{1-n} T^{-n} e^{\left(N-\sigma^{*}\right) t}\right) .
\end{aligned}
$$

The condition $0 \leq r_{p}(\mu) \leq F$ shall be an abbreviation for $\mu \in S_{p}, \operatorname{Im} r_{p}(\mu)=0$ and $\leq r_{p}(\mu) \leq F$. The Weyl asymptotic estimate (1) implies $\sum_{0 \leq r_{p}(\mu) \leq F} d_{p}^{*}(\mu)=O\left(F^{n}\right)=$ $O\left(T^{n}\right)$ and thereby

$$
L_{9}=O\left(t^{1-n} e^{\left(N-\sigma^{*}\right) t}\right)=O\left(T^{2 N} e^{\left(N-0^{*}\right) t}\right) .
$$

In order to get this estimation we have used $I m s^{-}(\mu) \leq 0$ and $\left|s^{-} .(\mu)-s\right| \geq T$. We can not use a similar consideration for $s^{+}(\mu)$ instead of $s^{-}(\mu)$. We get

$$
\left|t^{1-n} \sum_{0 \leq \sum_{p}(\mu) \leq F} 2 \kappa_{p} d_{p}^{*}(\mu) X\left(s^{-}(\mu), s, t\right)\right|=O\left(T^{2 N} e^{2 N\left(N-\sigma^{*}\right) t}\right)
$$

and the theorem is proved for $\tilde{S}^{\#}$ instead of $S^{\#}$. We have shown

$$
(-1)^{n-1} \Psi_{p}(s)=\Psi_{p}^{[t]}(s)+O\left(T^{2 N} e^{\left(N-\sigma^{\bullet}\right) t}\right)+t^{1-n} \sum_{0 \leq T_{p}(\mu) \leq F} 2 \kappa_{p} d_{p}^{*}(\mu) X\left(s^{+}(\mu), s, t\right)
$$

Next we consider

$$
L_{10}=t^{1-n} \sum_{0 \leq r_{p}(\mu) \leq G} 2 \kappa_{p} d_{p}^{*}(\mu) X\left(s^{+}(\mu), s, t\right)
$$

We easily see

$$
\left|L_{10}\right|=O\left(t^{1-n} \sum_{0 \leq r_{p}(\mu) \leq G} d_{p}^{*}(\mu) \frac{e^{\left(N-\sigma^{*}\right)(n-1) t}}{\left[\left(\sigma^{*}-N\right)^{2}+\left(T-r_{p}(\mu)^{2}\right]^{n / 2}\right.}\right)
$$

It follows

$$
\left|L_{10}\right|=O\left(t^{1-n} e^{\left(N-\sigma^{*}\right)(n-1) t}\left[1+\int_{1}^{G} \frac{1}{\left[\left(\sigma^{*}-N\right)^{2}+(T-r)^{2}\right]^{n / 2}} d \mathcal{N}_{p}(r)\right]\right)
$$


We apply Proposition 34. It holds $\mathcal{N}_{p}(r)=n_{p} r^{n}+\mathcal{R}_{p}(r)$ with $\left|\mathcal{R}_{p}(r)\right|=O\left(r^{n-1}\right)$. We split up the integral in (142). We first consider

$$
\begin{aligned}
L_{11} & =t^{1-n} \int_{1}^{G} \frac{1}{\left[\left(\sigma^{*}-N\right)^{2}+(T-r)^{2}\right]^{n / 2}} d\left(n_{p} r^{n}\right) \\
& =t^{1-n} \int_{1}^{G} \frac{n n_{p} r^{n-1}}{\left[\left(\sigma^{*}-N\right)^{2}+(T-r)^{2}\right]^{n / 2}} d r .
\end{aligned}
$$

It follows

$$
\begin{aligned}
\left|L_{11}\right| & =O\left(\frac{G^{n-1}}{t^{n-1}} \int_{1}^{G} \frac{1}{\left[\left(\sigma^{*}-N\right)^{2}+(T-r)^{2}\right]^{n / 2}} d r\right) \\
& =O\left(\frac{G^{n-1}}{t^{n-1}} \frac{1}{\left(\sigma^{*}-N\right)^{n}} \int_{1}^{G}\left[1+\left(\frac{T-r}{\sigma^{*}-N}\right)^{2}\right]^{-n / 2} d r\right) \\
& =O\left(\frac{G^{n-1}}{t^{n-1}} \frac{1}{\left(\sigma^{*}-N\right)^{n}} \int_{1}^{G}\left[1+\left(\frac{T-r}{\sigma^{*}-N}\right)^{2}\right]^{-1} d r\right) \\
& =O\left(\frac{G^{n-1}}{t^{n-1}} \frac{1}{\left(\sigma^{*}-N\right)^{n}} \int_{\frac{10}{\sigma^{+}-N}}^{\frac{T-1}{\sigma^{*}-N}} \frac{1}{1+\tilde{r}^{2}} d \tilde{r}\right)
\end{aligned}
$$

By Assumption $56 /\left(\right.$ ii) and (iii) we get $t\left(\sigma^{*}-N\right) \geq 1$ and thereby $\left|L_{11}\right|=O\left(G^{n-1}\right)=$ $O\left(T^{2 N}\right)$. The other term resulting from the decomposition $\mathcal{N}_{p}(r)=n_{p} r^{n}+\mathcal{R}_{p}(r)$ is

$$
L_{12}=t^{1-n} \int_{1}^{G}\left[\left(\sigma^{*}-N\right)^{2}+(T-r)^{2}\right]^{-n / 2} d \mathcal{R}_{p}(r) .
$$

The integrand $\left[\left(\sigma^{*}-N\right)^{2}+(T-r)^{2}\right]^{-n / 2}$ is monotonically encreasing for $0 \leq r \leq G$. We obtain $L_{12}=O\left(t^{1-n} \int_{1}^{G} d \mathcal{R}_{p}(r)\right)$. Using partial integration, it follows $L_{12}=O\left(G^{n-1} / t^{n-1}\right)$ and thereby $L_{12}=O\left(T^{2 N}\right)$. Using (142), we get

$$
\left|t^{1-n} \sum_{0 \leq r p(\mu) \leq G} X\left(s^{+}(\mu), s, t\right)\right|=O\left(T^{2 N} e^{\left(N-\sigma^{*}\right) t}\right) .
$$

Thereby the proof of Proposition 57 is completed

Proposition 58: We suppose (108) - (117), Assumption 47 and 56. Then it holds $\Psi_{p}\left(\sigma^{*}+i T\right)=(-1)^{n-1} \Psi_{p}^{[t]}(s)+O\left(T^{2 N} e^{\left(N-\sigma^{*}\right) t}\right)+O\left(e^{\left(N-\sigma^{*}\right) t}\left|\Psi_{p}^{[t]}\left(\sigma_{1}+i T\right)\right|\right)$.

Proof: It holds

$$
\left|e^{k\left(s^{+}(\mu)-s\right) t}\right|=e^{k\left(N-\sigma^{*}\right) t} \leq e^{\left(N-0^{*}\right) t}
$$


with $k \in \mathbf{N}$. It follows

$$
\left|\left[e^{\left(\bullet^{+}(\mu)-\rho\right) t}-e^{2\left(\bullet^{+}(\mu)-\rho\right) t}\right]^{n-1}\right| \leq 2^{n-1} e^{\left(N-\sigma^{*}\right) t}
$$

and thereby

$$
\begin{aligned}
t^{1-n} & \sum_{\left|\tau_{p}(\mu)-T\right| \leq 10} d_{p}^{*}(\mu) X\left(s^{+}(\mu), s, t\right) \\
= & t^{1-n} 2^{n-1} \vartheta e^{\left(N-\sigma^{*}\right) t} \sum_{\left|r_{p}(\mu)-T\right| \leq 10} \frac{d_{p}^{*}(\mu)}{\left[\left(\sigma^{*}-N\right)^{2}+\left(T-r_{p}(\mu)\right)^{2}\right]^{n / 2}}
\end{aligned}
$$

with $|\vartheta| \leq 1$. Assumption 56/(ii) implies

$$
\begin{aligned}
\frac{1}{t^{n-1}} \frac{1}{\left[\left(\sigma^{*}-N\right)^{2}+\left(T-r_{p}(\mu)\right)^{2}\right]^{n / 2}} & \leq \frac{\left(\sigma_{1}-N\right)^{n-1}}{\left[\left(\sigma_{1}-N\right)^{2}+\left(T-r_{p}(\mu)\right)^{2}\right]^{n / 2}} \\
& \leq \frac{\sigma_{1}-N}{\left[\left(\sigma_{1}-N\right)^{2}+\left(T-r_{p}(\mu)\right)^{2}\right]} .
\end{aligned}
$$

We apply Proposition 57 and we get with a number $\vartheta_{1}$ with $\left|\vartheta_{1}\right| \leq 1$ the equation

$$
\begin{aligned}
(-1)^{n-1} \Psi_{p}(s)= & \Psi_{p}^{[t]}(s)+O\left(T^{2 N} e^{\left(N-\sigma^{*}\right) t}\right) \\
& +2^{n-1} \vartheta_{1} e^{\left(N-\sigma^{*}\right) t} \\
& \times \sum_{\left|r_{p}(\mu)-T\right| \leq 10} 2 \kappa_{p} d_{p}^{*}(\mu) \frac{\sigma_{1}-N}{\left[\left(\sigma_{1}-N\right)^{2}+\left(T-r_{p}(\mu)\right)^{2}\right]}
\end{aligned}
$$

for $\sigma_{1} \leq \sigma^{*} \leq 4 N$. We remark that by Proposition 34 we can use $\sum_{\left|r_{p}(\mu)-T\right| \leq 1}$ as well as $\sum_{\left|r_{p}(\mu)-T\right| \leq 10}$ in the equations above. Further on, by Proposition 40 we obtain

$$
\Psi_{p}\left(\sigma_{1}+i T\right)=O\left(T^{n-1}\right)+\kappa_{p} \sum_{\left|r_{p}(\mu)-T\right| \leq 10} d_{p}^{*}(\mu) \frac{1}{\left(\sigma_{1}-N\right)+i\left(T-r_{p}(\mu)\right)} .
$$

We get

$$
R e \Psi_{p}\left(\sigma_{1}+i T\right)=O\left(T^{n-1}\right)+\kappa_{p} \sum_{\left|r_{p}(\mu)-T\right| \leq 10} d_{p}^{*}(\mu) \frac{\sigma_{1}-N}{\left(\sigma_{1}-N\right)^{2}+\left(T-r_{p}(\mu)\right)^{2}} .
$$

Equation (146) implies

$$
\begin{aligned}
\operatorname{Re} \Psi_{p}\left(\sigma_{1}+i T\right)= & \operatorname{Re}\left[(-1)^{n-1} \Psi_{p}^{[t]}\left(\sigma_{1}+i T\right)\right]+O\left(T^{2 N} e^{\left(N-\sigma_{1}\right) t}\right) \\
& +(-1)^{n-1} 2^{n-1} \vartheta_{1} e^{\left(N-\sigma_{1}\right) t} \cdot \\
& \times \sum_{\left|r_{p}(\mu)-T\right| \leq 10} 2 \kappa_{p} d_{p}^{*}(\mu) \frac{\sigma_{1}-N}{\left(\sigma_{1}-N\right)^{2}+\left(T-r_{p}(\mu)\right)^{2}}
\end{aligned}
$$

with $\left|\vartheta_{1}\right| \leq 2$. Using (147) and (148) it follows

$$
\begin{aligned}
\operatorname{Re} \Psi_{p}^{[l]}(s)= & {\left[1+(-1)^{n-1} \cdot 2^{n-1} \vartheta_{1} \frac{1}{e} \kappa_{p}\right] } \\
& \times \sum_{\left|r_{p}(\mu)-T\right| \leq 10} \frac{d_{p}^{*}(\mu)\left(\sigma_{1}-N\right)}{\left(\sigma_{1}-N\right)^{2}+\left(T-r_{p}(\mu)\right)^{2}}+O\left(T^{2 N}\right) .
\end{aligned}
$$


We get

$$
\sum_{\left|r_{p}(\mu)-T\right| \leq 10} d_{p}^{*}(\mu) \frac{\sigma_{1}-N}{\left(\sigma_{1}-N\right)^{2}+\left(T-r_{p}(\mu)\right)^{2}}=O\left(\left|\Psi_{p}^{[t]}\left(\sigma_{1}+i T\right)\right|\right)+O\left(T^{2 N}\right) .
$$

The combination of the last equation and equation(146) proves the assertion of the theorem 1

Proposition 59: We suppose (108) - (117), Assumption $\$ 7$ and 56. Then it holds

$$
\begin{aligned}
\arg Z_{p}(N+i T)= & -I m\left[\sum_{\omega \in \Omega} \Lambda_{p}^{t}(\omega) \frac{1}{l(\omega)} e^{-l(\omega)\left(\sigma_{1}+i T\right)}\right] \\
& +O\left(\frac{T^{n-1}}{t}\right)+O\left(\frac{1}{t}\left|\sum_{\omega \in \Omega} \Lambda_{p}^{t}(\omega) e^{-l(\omega)\left(\sigma_{1}+i T\right)}\right|\right) .
\end{aligned}
$$

Proof: We have

$$
\begin{aligned}
\arg Z_{p}(N+i T)= & -\int_{N}^{4 N} I m \Psi_{p}(\sigma+i T) d \sigma+\arg Z_{p}(4 N+i T) \\
= & -\int_{N}^{4 N} I m \Psi_{p}(\sigma+i T) d \sigma+O(1) \\
= & -\int_{\sigma_{1}}^{4 N} I m \Psi_{p}(\sigma+i T) d \sigma-\left(\sigma_{1}-N\right) \operatorname{Im} \Psi_{p}\left(\sigma_{1}+i T\right) \\
& +\int_{N}^{\sigma_{1}} \operatorname{Im}\left[\Psi_{p}\left(\sigma_{1}+i T\right)-\Psi_{p}(\sigma+i T)\right] d \sigma+O(1) .
\end{aligned}
$$

We consider the terms on the right-hand side.

1) We first consider $J_{1}=\int_{\sigma_{1}}^{4 N} I m \Psi_{p}(\sigma+i T) d \sigma$. Proposition 58 gives

$$
\begin{aligned}
J_{1}= & (-1)^{n-1} \int_{\sigma_{1}}^{4 N} I m \Psi_{p}^{[t]}(\sigma+i T) d \sigma \\
& +O\left(\left|\Psi_{p}^{[t]}\left(\sigma_{1}+i T\right)\right|\right) \int_{\sigma_{1}} e^{(N-\sigma) t} d \sigma+O\left(T^{2 N} \int_{\sigma_{1}}^{4 N} e^{(N-\sigma) t} d \sigma\right) \\
= & (-1)^{n} \kappa_{p} I m \sum_{\omega \in \Omega} \Lambda_{p}^{t}(\omega) \frac{1}{l(\omega)} e^{-l(\omega)\left(\sigma_{1}+i T\right)}+O(1) \\
& +O\left(\left|\sum_{\omega \in \Omega} \Lambda_{p}^{t}(\omega) e^{-l(\omega)\left(\sigma_{1}+i T\right)}\right| \frac{1}{t} e^{\left(N-\sigma_{1}\right) t}\right)+O\left(T^{2 N} \frac{1}{t} e^{\left(N-\sigma_{1}\right) t}\right) .
\end{aligned}
$$

It follows

$$
\begin{aligned}
J_{1}= & (-1)^{n} \kappa_{p} I m \sum_{\omega \in \Omega} \Lambda_{p}^{t}(\omega) \frac{1}{l(\omega)} e^{-l(\omega)\left(\sigma_{1}+i T\right)}+O(1) \\
& +O\left(\frac{1}{t}\left|\sum_{\omega \in \Omega} \Lambda_{p}^{t}(\omega) e^{-l(\omega)\left(\sigma_{1}+i T\right)}\right|\right)+O\left(T^{2 N} \frac{1}{t}\right)
\end{aligned}
$$


2) We consider $J_{2}=-\left(\sigma_{1}-N\right) \operatorname{Im} \Psi_{p}\left(\sigma_{1}+i T\right)$. By Proposition 58 we obtain

$$
\begin{aligned}
\left|J_{2}\right| & =O\left(\left(\sigma_{1}+i T\right)\left|\Psi_{p}\left(\sigma_{1}+i T\right)\right|\right) \\
& =O\left(\frac{1}{t}\left|\sum_{\omega \in \mathbb{\Omega}} \Lambda_{p}^{t}(\omega) e^{-l(\omega)\left(\sigma_{1}+i T\right)}\right|\right)+O\left(T^{2 N} \frac{1}{t}\right) .
\end{aligned}
$$

3) We consider $J_{3}=\int_{N}^{\sigma_{1}} \operatorname{Im}\left[\Psi_{p}\left(\sigma_{1}+i T\right)-\Psi_{p}(\sigma+i T)\right] d \sigma$. Proposition 40 yields

$$
\begin{aligned}
& \operatorname{Im}\left[\Psi_{p}\left(\sigma_{1}+i T\right)-\Psi_{p}(\sigma+i T)\right]-O\left(T^{n-1}\right) \\
& =+\kappa_{p} \sum_{\left|r_{p}(\mu)-T\right| \leq 10} d_{p}^{*}(\mu) \operatorname{Im}\left(\frac{1}{\sigma_{1}-N+i\left(T-r_{p}(\mu)\right)}-\frac{1}{\sigma-N+i\left(T-r_{p}(\mu)\right)}\right) \\
& =\kappa_{p} \sum_{\left|r_{p}(\mu)-T\right| \leq 10} d_{p}^{*}(\mu)\left(\frac{-\left(T-r_{p}(\mu)\right)}{\left(\sigma_{1}-N\right)^{2}+\left(T-r_{p}(\mu)\right)^{2}}+\frac{T-r_{p}(\mu)}{(\sigma-N)^{2}+\left(T-r_{p}(\mu)\right)^{2}}\right) \\
& =\kappa_{p} \sum_{\left|r_{p}(\mu)-T\right| \leq 10} d_{p}^{*}(\mu) \frac{\left[T-r_{p}(\mu)\right]\left[\left(\sigma_{1}-N\right)^{2}-(\sigma-N)^{2}\right]}{\left[\left(\sigma_{1}-N\right)^{2}+\left(T-r_{p}(\mu)\right)^{2}\right]\left[(\sigma-N)^{2}+\left(T-r_{p}(\mu)\right)^{2}\right]} \\
& \leq \kappa_{p} \sum_{\left|r_{p}(\mu)-T\right| \leq 10} d_{p}^{*}(\mu) \frac{\left|T-r_{p}(\mu)\right|\left(\sigma_{1}-N\right)^{2}}{\left[\left(\sigma_{1}-N\right)^{2}+\left(T-r_{p}(\mu)\right)^{2}\right]\left[(\sigma-N)^{2}+\left(T-r_{p}(\mu)\right)^{2}\right]} .
\end{aligned}
$$

It follows

$$
\begin{aligned}
\left|J_{3}\right| \leq & \int_{N}^{\sigma_{1}} O\left(T^{n-1}\right) \\
& +\kappa_{p} \sum_{\left|r_{p}(\mu)-T\right| \leq 10} \frac{d_{p}^{*}(\mu)\left|T-r_{p}(\mu)\right|\left(\sigma_{1}-N\right)^{2}}{\left[\left(\sigma_{1}-N\right)^{2}+\left(T-r_{p}(\mu)\right)^{2}\right]\left[(\sigma-N)^{2}+\left(T-r_{p}(\mu)\right)^{2}\right]} d \sigma \\
\leq & O\left(T^{n-1}\left(\sigma_{1}-N\right)\right) \\
& +\kappa_{p} \sum_{\left|r_{p}(\mu)-T\right| \leq 10} d_{p}^{*}(\mu) \frac{\left(\sigma_{1}-N\right)^{2}}{\left(\sigma_{1}-N\right)^{2}+\left(T-r_{p}(\mu)\right)^{2}} \int_{N}^{\sigma_{1}} \frac{\left|T-r_{p}(\mu)\right|}{(\sigma-N)^{2}+\left(T-r_{p}(\mu)\right)^{2}} d \sigma \\
= & O\left(T^{n-1}\left(\sigma_{1}-N\right)\right)+O\left(\sum_{\left|r_{p}(\mu)-T\right| \leq 10} d_{p}^{*}(\mu) \frac{\left(\sigma_{1}-N\right)^{2}}{\left(\sigma_{1}-N\right)^{2}+\left(T-r_{p}(\mu)\right)^{2}}\right) \\
= & O\left(T^{n-1} \frac{1}{t}\right)+O\left(\frac{1}{t} \sum_{\left|r_{p}(\mu)-T\right| \leq 10} d_{p}^{*}(\mu) \frac{\sigma_{1}-N}{\left(\sigma_{1}-N\right)^{2}+\left(T-r_{p}(\mu)\right)^{2}}\right) .
\end{aligned}
$$

We apply (151) and get

$$
J_{3}=\dot{O}\left(T^{n-1} \frac{1}{t}\right)+O\left(\frac{1}{t}\left|\sum_{\omega \in \Omega} \Lambda_{p}^{t}(\omega) e^{-l(\omega) \bullet}\right|\right) .
$$

Summarizing (152) - (155) we obtain the assertion

Now we can give the 
Proof of Theorem B: We first suppose (113). Proposition 59 implies

$$
\begin{aligned}
\arg Z_{p}(N+i T)= & (-1)^{n} \sum_{\omega \in \Omega} \Lambda_{p}^{t}(\omega) \frac{1}{l(\omega)} e^{-l(\omega) \sigma_{1}} \sin (l(\omega) T) \\
& +O\left(\frac{1}{t}\left|\sum_{\omega \in \Omega} \Lambda_{p}^{t}(\omega) e^{-l(\omega)\left(\sigma_{1}+i T\right)}\right|\right)+O\left(\frac{T^{n-1}}{t}\right) \\
= & O\left(\frac{1}{t}\left|\sum_{\omega \in \Omega} \Lambda_{p}^{t}(\omega)\right| e^{-l(\omega) N}\right)+O\left(\frac{T^{n-1}}{t}\right) .
\end{aligned}
$$

Equation (119) implies $\Lambda_{p}^{t}(\omega)=O\left(\Lambda_{p}(\omega)\right)$ with an $O$-term not depending on $\omega \in \Omega$. Using (122), it follows

$$
\arg Z_{p}(N+i T)=O\left(\sum_{\substack{\omega \in \mathbb{n} \\ l(\omega) \leq 2(n-1) t}} \frac{1}{t} \Lambda_{p}(\omega) e^{-l(\omega) N}\right)+O\left(\frac{T^{n-1}}{t}\right)
$$

Proposition 5 implies

$$
\sum_{\substack{\omega \in \Omega \\ l(\omega) \leq 2(n-1) t}} \frac{1}{t} \Lambda_{p}(\omega) e^{-l(\omega) N}=O\left(e^{N 2(n-1) t}\right) .
$$

We now use $e^{t}=T^{1 / 7 N}$ (cf.Assumption 56/(i)) and get

$$
\arg Z_{p}(N+i T)=O\left(\frac{T^{4 N / 7}}{t}\right)+O\left(\frac{T^{n-1}}{t}\right)
$$

It follows

$$
\arg Z_{p}(N+i T)=O\left(\frac{T^{n-1}}{\ln T}\right)
$$

and thereby

$$
\left|\mathcal{R}_{p}(T)\right|=O\left(\frac{T^{n-1}}{\ln T}\right) .
$$

By right continuity this equation is valid for all $T$ and Theorem B is proved

\section{References}

[29] Christian, U.: Untersuchung Selbengscher Zetafunktionen. J. Math. Soc. Japan 41 (1989), $503-537$.

[30] Christian, U.: Zur Theorie Selberg'scher Zetafunktionen. Arch. Math. 54 (1990), 474 - 486.

[31] Efrat,I.: Selberg trace formula, rigidity, Weyl's law, and cusp forms. Ph. D. Theses. New York Univ. 1983.

[32] Fischer, J.: An approach to the Selberg trace formula via the Selberg zeta-function. Lect. Notes Math. 1253 (1987), 1 - 184. 
[33] Grosche, Chr.: Selbery supertrace formula for super Riemann surfaces, analytic properties of Selberg super zeta-functions and multiloop contributions for the fermionic string. Commun. Math. Phys. 133 (1990), 433 - 485.

[34] Huntley,J.: Spectral multiplicity on products of hyperbolic spaces. Proc. Amer. Math. Soc. $111(1991), 1$ - 12.

[35] Schuster, R.: Selbergsche Zetafunktion, Spektralabschätzungen, geodätische Doppeldifferentialformen und Gitterprobleme als Elemente der hyperbolischen Spektralgeometrie. Dissertation B. Universität Leipzig 1990.

[36] Schuster, R.: Spectral estimates for compact hyperbolic space forms and the Selberg zeta function for p-spectra. Part I. Z. Anal. Anw. 11 (1992), 343 - 358.

[37] Seeley, E.: An estimate near the boundary for the spectral function of the Laplace operator. Amer. J. Math. 102 (1980), 869 - 902.

Received 19.09.1993 